\title{
Difeomorfismos do plano com número de rotação de fins primos irracional
}

\author{
Diego Pereira Barboza
}

TESE APRESENTADA

$\mathrm{AO}$

Instituto de Matemática e Estatística

DA

Universidade de SÃo Paulo

PARA

OBTENÇÃO DO TÍTULO

$\mathrm{DE}$

Doutor EM CIÊNCIAS

Programa: Doutorado em Matemática Aplicada do IME-USP

Orientador: Prof. Dr. Salvador Addas Zanata

Durante o desenvolvimento deste trabalho o autor recebeu auxílio financeiro da CAPES

São Paulo, Dezembro de 2018 


\section{Resumo}

\section{BARBOZA D.P. Difeomorfismos do plano com número de rotação de fins pri-}

mos irracional. 2018. 72+vi f. Tese (Doutorado) - Instituto de Matemática e Estatística, Universidade de São Paulo, São Paulo, 2018.

O principal objetivo desta tese é estudar o número de rotação de fins primos de homeomorfismos planares que pertencem a uma classe de homeomorfismos $\mathcal{H}$. Tal número de rotação é devido à Carathéordory e semelhante à teoria de Poincaré para homeomorfismos do círculo. Para todo irracional $\alpha \in(0,1)$, denotando por $\rho(h, U)$ o número de rotação de fins primos de $h \in \mathcal{H}$ em $U$, com $U$ a bacia de repulsão do infinito, construiremos um homeomorfismo $h \in \mathcal{H}$ satisfazendo $\rho(h, U)=\alpha$ e que possui uma sela periódica com intersecção homoclínica transversal em $\partial U$. Além disso, quando $h$ é de classe $C^{2}$ e $\operatorname{det}\left(\left.D h\right|_{x}\right)<1$ em todo ponto, mostraremos que existe ponto periódico acessível em $\partial U$ se, e somente se, $\rho(h, U)$ é racional.

Também será provado que, quando $h$ é uma ferradura de Smale, o número de rotação $\rho(h, U)$ é racional. Finalizando, provaremos que se for possível a existência de um difeomorfismo $C^{r}, r \geq 1$, em um conjunto genérico a ser definido, com $\partial U=\overline{\mathcal{W}^{u}(p)}$ para $p$ uma sela homoclínica com intersecção transversal e tal que o número de rotação $\rho(h, U)$ é irracional, necessariamente, $h$ deve satisfazer uma propriedade que não é válida para ferraduras de Smale.

Palavras-chave: Difeomorfismos Planares; Número de Rotação; Teoria de Fins Primos. 


\section{Abstract}

BARBOZA D.P. Diffeomorphisms of the plane with irrational prime ends rotation number 2018. 72+vi pp. PhD thesis- Instituto de Matemática e Estatística, Universidade de São Paulo, São Paulo, 2018.

The main objective of this thesis is to study the prime ends rotation number of planar homeomorphisms belonging to a class of homeomorphisms $\mathcal{H}$. Such rotation number is due to Carathéordory and similar to the Poincaré's theory of homeomorphisms of the circle. For all irrational $\alpha \in(0,1)$, denoting by $\rho(h, U)$ the prime end rotation number of $h \in \mathcal{H}$ in $U$, with $U$ the infinity repulsion basin, we will construct a homeomorphism $h \in \mathcal{H}$ satisfying $\rho(h, U)=\alpha$ and having a homoclinic saddle with transverse intersection in $\partial U$. Also, when $h$ is class $C^{2}$ and $\operatorname{det}\left(\left.D h\right|_{x}\right)<1$ at every point, we will show that there is accessible periodic point in $\partial U$ if, and only if, $\rho(h, U)$ is rational.

It will also be proved that when $h$ is a Smale's horseshoe, the rotation number $\rho(h, U)$ is rational. To conclude, we will prove that if there exists a $C^{r}$-diffeomorphism, in a generic set to be defined, with $\partial U=\overline{\mathcal{W}^{u}(p)}$ for a saddle point $p$ with transverse homoclinal intersection and such that the rotation number $\rho(h, U)$ is irrational, then $h$ must satisfy a property that is not valid for Smale's horseshoes.

Keywords: Planar Diffeomorphisms; Rotation Number; Prime Ends Theory. 


\section{Sumário}

1 Introdução $\quad 7$

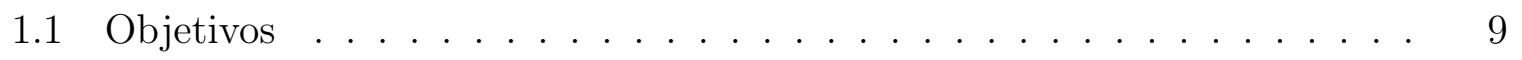

1.2 Resultados Obtidos . . . . . . . . . . . . . . . . . . . 11

2 Preliminares $\quad 13$

2.1 Conceitos Básicos e Definições . . . . . . . . . . . . . . . . . 13

2.2 Uma Propriedade dos continuos . . . . . . . . . . . . . . . . 18

2.3 Número de Rotação de Homeomorfismos do Círculo . . . . . . . . . . . 21

2.4 Teoria de Fins Primos f . . . . . . . . . . . . . 25

3 Alguns Resultados $1 \quad 31$

3.1 Sobre à Classe de Homeomorfismos $\mathcal{H} \ldots \ldots$. . . . . . . . . . . 31

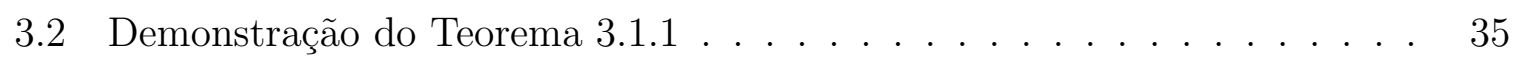

3.3 O exemplo de Matsumoto . . . . . . . . . . . . . . . 45

3.4 O exemplo de Bernado e Salvador . . . . . . . . . . . . . . . 48

3.5 Demonstração do Teorema $3.1 .4 \ldots \ldots$. . . . . . . . . . . 49

4 Alguns Resultados 2 


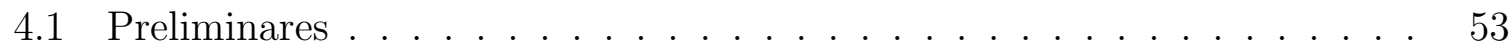

4.2 Número de Rotação da Ferradura de Smale . . . . . . . . . . . . . . . . . 57

4.3 Consequências do Número de Rotação de Fins Primos $\rho(h, U)$ Irracional. 64 


\section{Lista de Figuras}

2.1 Informações dos conjuntos $\Sigma$ e $M \ldots \ldots \ldots \ldots$

3.1 O conjunto $\Sigma$ visto como um subconjunto da esfera. . . . . . . . . . 33

3.2 Se $x \neq h(x)$ em $\mathbb{R}^{2}$, então $\mathfrak{p} \neq \tilde{h}(\mathfrak{p})$ em $\mathbb{P}(U) \ldots \ldots \ldots \ldots$

3.3 Informações na compactificação de fins primos de $U$. . . . . . . . . . . . 41

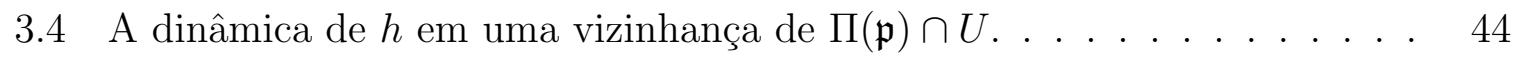

3.5 A dinâmica de $h$ perto de $\Pi(\mathfrak{p}) \cap U, p_{0} \in \Pi(\mathfrak{p}) \cap U$ e $V$ uma vizinhaça aberta de $p . \ldots \ldots \ldots \ldots \ldots \ldots 4 \ldots \ldots \ldots$

3.6 Em vermelho, temos os "cabelos" de $\Sigma$ com origem nos pontos do conjunto minimal $C_{\alpha}$ e que estão se enrolando em torno do atrator do exemplo de Bernado e Salvador. . . . . . . . . . . . . . . . . . . . . . . . 51

4.1 Informações na compactificação de fins primos de $U$. . . . . . . . . . 56

4.2 Exemplo em que a intersecção $h(Q) \cap Q$ possui 3 retângulos . . . . . . . 58

4.3 Informaçõs de $Q \cap h(Q)$ no plano. . . . . . . . . . . . . . . . 61

4.4 Semi-reta $l$ acessando o ponto $a \in \Gamma \ldots \ldots \ldots$. . . . . . . . 63 


\section{Capítulo 1}

\section{Introdução}

O conceito de número de rotação de um homeomorfismo do círculo que preserva orientação foi introduzido por Henri Poincaré em [Po52], e se mostrou uma ferramenta muito útil para se descrever a dinâmica de tais aplicações. A dinâmica desses homeomorfismos é topologicamente simples e pode ser caracterizada pelo número de rotação.

Poincaré percebeu que, dado um homeomorfismo do círculo que preserva orientação, $h: S^{1} \rightarrow S^{1}$, podemos considerar um ponto $x \in S^{1}$ qualquer e obter que as médias $\frac{H^{n}(\tilde{x})-\tilde{x}}{n}$ convergem para um mesmo número, com $H: \mathbb{R} \rightarrow \mathbb{R}$ um levantamento para o recobrimento universal de $h$ e $\tilde{x}$ satisfazendo $\pi(\tilde{x})=x$ onde $\pi: \mathbb{R} \rightarrow S^{1}$ é a aplicação de recobrimento. Este número é chamado de número de rotação de $\tilde{h}$. O seguinte resultado, conhecido como Teorema de Poincaré, pode ser encontrado em [KH99].

O número de rotação de um homeomorfismo do círculo $S^{1}$ que preserva orientação é racional se, e somente se, este homeomorfismo possuir um ponto periódico cujo período é igual ao denominador de tal racional, pensando na fração irredutível.

Com o objetivo de estudar a dinâmica de um homeomorfismo $h$ do plano que preserva orientação na fronteira de um aberto $U$ conexo, simplesmente conexo e invariante por $h$, é natural tentar definir um invariante similar à tal número de rotação e tentar aplicar o resultado acima. Embora $U$ seja topologicamente um disco aberto, a fronteira de $U$ pode 
não ser topologicamente um círculo e pode possuir uma estrutura muito complicada, por exemplo, a fronteira de $U$ pode não ser localmente conexa em nenhum ponto.

A teoria de fins primos, desenvolvida por Carathéodory [Ca13], fornece uma compactificação da região $U$ adicionando um conjunto $\mathbb{P}(U)$ chamado de conjunto de fins primos de $U$, tal que, com uma topologia que surge naturalmente da construção, o conjunto dos fins primos é homeomorfo a um círculo e o conjunto $U^{*}=U \cup \mathbb{P}(U)$ é homeomorfo ao disco unitário fechado.

Desse modo, o homeomorfismo $h$ pode ser estendido para um homeomorfismo $\tilde{h}$ de $U^{*}$, cuja restrição $\left.\tilde{h}\right|_{\mathbb{P}(U)}$ induz um homeomorfismo $h^{\prime}: S^{1} \rightarrow S^{1}$ que preserva orientação. Portanto, podemos definir para cada homeomorfismo do plano $h$ que preserva orientação e cada aberto $U$, conexo, simplesmente conexo e invariante por $h$, um número de rotação de fins primos $\rho(h, U)$ como sendo o número de rotação de Poincaré de $h^{\prime}$. A compreensão da relação do número de rotação de $h^{\prime}$ com a dinâmica de $h$ na fronteira de $U$ é um problema sutil que tem sido estudado em vários trabalhos.

O Teorema de Poincaré para homeomorfismos do círculo mostra como o número de rotação clássico está relacionado com a existência de órbitas periódicas. Para os homeomorfismos do plano $h$ e os domínios simplesmente conexos $U$, já foi observado por Cartwright e Littlewood em [CL51] que o número de rotação $\rho(h, U)$ ser racional não é suficiente para garantir a existência de órbitas periódicas na fronteira de $U$. No entanto, com algumas hipóteses adicionais ao homeomorfismo ou na região $U$, é possível obter algum tipo de conexão entre as duas noções. Por exemplo, no caso em que $h$ preserva área a situação é diferente, como foi mostrado por Cartwright e Littleword em [CL51]:

\section{Se h preserva área e o número de rotação de fins primos \\ é racional, então existe ponto periódico em $\partial U$.}

Resultados semelhantes, obtendo pontos periódicos a partir de um número de rotação de fins primos racional, foram provados por vários autores, por exemplo em [AY92, BG91, BG92, BaK98]. A afirmação inversa questiona se a existência de pontos periódicos implica que o número de rotação de fins primos é racional. Neste caso, alguns resultados foram 
obtidos em [KLN15] mas, em geral, isto não é verdade, como foi provado por Matsumoto em [Mat13].

Em [KLN15], Koropecki, Le Calvez e Nassiri deram alguma luz sobre como é a dinâmica induzida na fronteira de $U$ quando o número de rotação de fins primos é irracional e o homeomorfismo satisfaz algumas hipóteses não-errantes em uma vizinhança de $\partial U$, que é satisfeita, por exemplo, quando o homeomorfismo preserva área. Como um corolário, eles provam que para homeomorfismos no plano, não pode existir pontos periódicos na fronteira de $U$, desde que o homeomorfismo satisfaça esta hipótese extra.

No caso em que o número de rotação $\rho(h, U)$ é irracional, alguns exemplos mostram que, mesmo quando $h$ preserva área, pode existir ponto fixo em $\partial U$ (ver exemplos 9.5 e 9.6 em [KNL15]). No entanto, estes exemplos são bem específicos e possuem apenas um ponto fixo e não possuem outros pontos periódicos.

\subsection{Objetivos}

Nesta tese, iremos trabalhar com uma classe específica de homeomorfismos do plano que será denotada por $\mathcal{H}$. A seguir, definiremos o que significa o infinito ser um ponto repulsor e definiremos também a classe de homeomorfismos $\mathcal{H}$.

Definição 1.1.1. O infinito é chamado de um ponto repulsor para um homeomorfismo $h: \mathbb{R}^{2} \rightarrow \mathbb{R}^{2}$ quando existirem curvas de Jordan $\gamma$, arbitrariamente distantes da origem que enlaçam a origem, tais que $h(\gamma)$ está contida no disco D limitado pela curva $\gamma$.

Definição 1.1.2. Considere $h$ um homeomorfismo do plano e suponha que $h$ preserva orientação. Diremos que $h$ pertence ao conjunto $\mathcal{H}$ quando:

1. O infinito é um ponto repulsor para $h$ e existe um ponto fixo atrator $p$ para $h$;

2. O homeomorfismo $h$ é dissipativo, isto é, para todo aberto $D \subset \mathbb{R}^{2}$, a área de $h^{n}(D) \rightarrow 0$, quando $n \rightarrow \infty ;$

3. A região de repulsão do infinito, $U=U_{\infty}$, é um subconjunto próprio de $\mathbb{R}^{2} \backslash 0$. 
Sem perda de generalidade, iremos supor que, para todo $h \in \mathcal{H}$, o ponto fixo atrator $p$ na definição 1.1.2 pode ser tomado como sendo a origem. Denotando por $U$ a bacia de repulsão do infinito por $h$, é possível mostrar a existência de um conjunto conexo e compacto $\Sigma=\partial U \subset \mathbb{R}^{2}$, que contém a origem, é um atrator global para $h$ e possui interior vazio. Além disso, definindo $h(\infty)=\infty$, podemos considerar a extensão natural de $h$ para a esfera de Riemann $S^{2}=\mathbb{R}^{2} \cup\{\infty\}$, para obtermos que $U$ é um domínio conexo, simplesmente conexo e invariante de $S^{2}$. Desse modo, a teoria de fins primos desenvolvida por Carathéodory pode ser aplicada. Pretendemos fazer um link entre a teoria do número de rotação de fins primos associado ao conjunto $U$ com a existência de órbitas periódicas. Assim, estudaremos se o número de rotação de alguma forma liga o comportamento dinâmico local e global.

Dado $h \in \mathcal{H}$, quando o número de rotação $\rho(h, U)$ é irracional, alguns resultados foram obtidos em [COR10] e [Mat13]. Em [COR10], os autores forneceram uma hipótese suficiente para que a origem seja o único ponto periódico de $h$. Em [Mat13], Matsumoto provou o seguinte Teorema:

Teorema 1.1.1. Para quaisquer números reais $\alpha$ e $\beta$, existe um homeomorfismo $h \in \mathcal{H}$ tal que $\rho\left(h, U_{\infty}\right)=\alpha$ e $\rho\left(h, U_{0}\right)=\beta$, onde $U_{\infty}$ é a bacia de repulsão de $\infty$ e $U_{0}$ a bacia de atração de 0 por $h$.

Este resultado mostra que existem homeomorfismos $h \in \mathcal{H}$ com número de rotação $\rho(h, U)$ irracional e que possuem órbitas periódicas em $\partial U$. Dito de outro modo, a existência de órbitas periódicas não implica que o número de rotação $\rho(h, U)$ é racional. Nos próximos capítulos, apresentaremos detalhadamente estes resultados.

Tendo em vista o resultado de Matsumoto, o principal objetivo desta tese é estudar a seguinte pergunta.

Pergunta 1: Dado $h \in \mathcal{H}$ um difeomorfismo de classe $C^{1}$, se o número de rotação $\rho(h, U)$ for irracional, é possível existir um ponto periódico de sela com intersecção homoclínica $C^{1}$-transversa na fronteira de $U$ ? 


\section{$1.2 \quad$ Resultados Obtidos}

No capítulo 2 apresentaremos os resultados básicos para o entendimento da teoria, definiremos o número de rotação de um homeomorfismo do círculo, o que é a compactificação de fins primos de um aberto conexo, simplesmente conexo e invariante do plano ou da esfera e exibiremos alguns resultados importantes destas teorias.

O capítulo 3 é dedicado os estudo detalhado da classe de homeomorfismos $\mathcal{H}$ e a exposição dos principais resultados de [COR10] e [Mat13] que motivaram esta tese. Ainda no capítulo 3, provaremos seguintes resultados:

Teorema 1.2.1. Para todo $h \in \mathcal{H}$ com $\rho(h, U)$ racional, existe ponto periódico diferente da origem em $\partial U$. Adicionalmente, supondo que $h$ é um difeomorfismo de classe $C^{2} e$ que $\operatorname{det}\left(\left.D h\right|_{x}\right)<1$ para todo $x \in \mathbb{R}^{2}$, então este ponto periódico é acessível.

No próximo Teorema, provaremos que existe $h \in \mathcal{H}$ que responde positivamente à Pergunta 1. Este $h$ não é um difeomorfismo, mas é um difeomorfismo local em disco topológico que contém os pontos periódicos de sela.

Teorema 1.2.2. Dado $\alpha \in(0,1)$ irracional, existe $h \in \mathcal{H}$ com número de rotação $\rho(h, U)=\alpha$ e tal que $\partial U$ possui um ponto $p$ de sela com intersecção homoclínica.

Tendo em vista o Teorema anterior, no capítulo 4 estudamos a Pergunta 1 com a hipótese adicional de que $\Sigma=\overline{W^{u}(p)}$, para $p \in \Sigma$ um ponto de sela com intersecção homoclínica. Neste caminho, provamos o seguinte resultado que responde negativamente a pergunta Pergunta 1 quando $h$ é uma ferradura de Smale generalizada:

Teorema 1.2.3. Dado $h \in \Sigma$, se $h$ é uma ferradura de Smale generalizada, então o número de rotação $\rho(h, U) \in \mathbb{Q}$.

A seguir, iremos apresentar algumas definições necessárias para o enunciado do próximo Teorema.

Um endcut de $U$ em é um arco $\gamma:[0,1) \rightarrow U$ tal que $\lim _{t \rightarrow 1} \gamma(t)=x$, para algum $x \in \partial U$ (tal que $\gamma$ estende continuamente para um arco $[0,1] \rightarrow \bar{U} \operatorname{com} \gamma(1)=x \in \partial U$ ). 
Um ponto $a \in \partial U$ é dito acessivel (por $U$ ) se é um ponto final para algum endcut. Neste caso, diremos que o endcut $\gamma$ acessa o ponto $a$.

Um crosscut $c$ de $U$ é a imagem de um arco $\gamma:(0,1) \rightarrow U$ que pode ser estendido para um arco $\bar{\gamma}:[0,1] \rightarrow \bar{U}$ tal que seus dois pontos finais são pontos acessíveis diferentes em $\partial U$. No Lema 2.2.2 provaremos que um crosscut divide $U$ em duas regiões que são topologicamente discos abertos e chamadas de cross-sections.

Finalizando o capítulo 4, provaremos que se for possível a existência de um difeomorfismo $C^{1}, r \geq 1$, genérico num certo sentido a ser precisado, com $\Sigma=\overline{\mathcal{W}^{u}(p)}$ e tal que o número de rotação $\rho(h, U)$ é irracional, necessariamente $h$ deve satisfazer a propriedade do próximo Teorema, onde $\sigma_{a}$ é qualquer crosscut com pontos finais nos pontos $a$ e $h(a)$, $D_{1}$ e $D_{2}$ as componentes conexas abertas de $\left(\Sigma \cup \sigma_{a}\right)^{c}$ e $K_{i}^{a}=\partial D_{i} \backslash \sigma_{a}, \operatorname{com} i=1,2$.

Teorema 1.2.4. Se o número de rotação $\rho(h, U)$ é irracional então, para todo ponto $a \in \Sigma$ acessivel, temos que $K_{1}^{a}=K_{2}^{a}=\Sigma$. 


\section{Capítulo 2}

\section{Preliminares}

Ao longo deste capítulo, será feita uma breve exposição de conceitos e resultados preliminares cujo entendimento é primordial para a compreensão dos resultados obtidos nesta tese. Entre tais conceitos, definiremos o número de rotação de um homeomorfismo do círculo, o que é a compactificação de fins primos de um aberto conexo e simplesmente conexo do plano ou da esfera e exibiremos alguns resultados importantes destas teorias.

\subsection{Conceitos Básicos e Definições}

Nesta seção veremos conceitos básicos e definições que serão utilizados no decorrer desta tese. De um modo geral sempre consideramos como espaço ambiente o plano $\mathbb{R}^{2}$. Em alguns momentos exigimos que o espaço ambiente seja a esfera de Riemann $S^{2}=\mathbb{R}^{2} \cup\{\infty\}$.

Consideramos $h: \mathbb{R}^{2} \rightarrow \mathbb{R}^{2}$ um homeomorfismo. Um ponto $x \in \mathbb{R}^{2}$ é periódico quando existir um inteiro $p>0$ tal que $h^{p}(x)=x$, o menor $p>0$ com tal propriedade é o período de $x$. Quando $p=1, x$ é chamado de ponto fixo. Denotaremos o conjunto de todos os pontos periódicos de $h$ por $\operatorname{Per}(h)$. Um conjunto $A \subset \mathbb{R}^{2}$ é invariante por $h$, quando $h(A)=A$. Um conjunto compacto $A$ é chamando de conjunto minimal quando todas as órbitas contidas em $A$ são densas em $A$. As operações topológicas de fecho, fronteira e 
interior de um conjunto, serão denotadas por $\bar{A}, \partial A$ e int $A$ e entendidas com relação ao plano ou a esfera.

Um conjunto $A$ contido no plano ou na esfera é topologicamente um disco aberto (resp. fechado) se é homeomorfo ao disco aberto (resp. fechado) unitário em $\mathbb{R}^{2}$. Similarmente, um anel topológico aberto (resp. fechado) é um conjunto homeomorfo ao anel $S^{1} \times(0,1)$ (resp. $\left.S^{1} \times[0,1]\right)$.

Dois homeomorfismos $h$ e $g$ são conjugados se existe um terceiro homeomorfismo $f$ tal que $h \circ f=f \circ g$. Dizemos que um conjunto $S \subset \mathbb{R}^{2}$ é um continuo, caso $S$ seja conexo, compacto e não-vazio. Se $S$ contém mais de um ponto, dizemos que $S$ é um continuo não-degenerado. Finalmente, se $T \subset S$ é um continuo, dizemos que $T$ é um subcontinuo de $S$.

Considere $h: M \rightarrow M$ um difeomorfismo de classe $C^{1}$ onde $M$ é uma superfície e seja $p \in M$ um ponto fixo de $h$. Denotando por $D h(p)$ a matriz Jacobiana de $h$ no ponto $p$, temos as seguintes possibilidades:

- um autovalor de $D h(p)$ é igual a 1 ou -1: o ponto $p$ é chamado ponto degenerado;

- os autovalores de $D h(p)$ são dois números complexos diferentes de 1 e ambos com módulo 1: o ponto $p$ é ponto elíptico;

- os autovalores de $D h(p)$ são ambos de módulo menor que 1: o ponto $p$ é chamado atrator;

- os autovalores de $D h(p)$ são ambos de módulo maior que 1: o ponto $p$ é chamado fonte;

- os autovalores $\lambda$ e $\sigma$ de $D h(p)$ são reais e satisfazem $0<|\lambda|<1<|\sigma|$ : o ponto $p$ é chamado de ponto de sela.

Se os dois autovalores de $D h(p)$ tem módulo diferente de 1, $p$ é chamado de ponto fixo hiperbólico. Por simplicidade, no último caso, assumimos que $0<\lambda<1<\sigma$. Vamos 
agora enunciar um Teorema importante, cuja demonstração pode ser encontrada em [Ha82].

Teorema 2.1.1. (Hartman,1964). Seja $h$ um difeomorfismo de classe $C^{r}, r \geq 1$, em uma superfície $M$ e $p \in M$ um ponto fixo de sela para $h$. Então $h$ admite coordenadas linearizantes em uma vizinhança de p.

Aqui, entendemos por coordenadas linearizantes a uma mudança de coordenadas que torne a função linear nas novas coordenadas. Assumiremos aqui que em uma vizinhança $U$ de $p, x_{1}$ e $x_{2}$ são coordenadas tais que $p=(0,0)$ e tal que

$$
\tilde{h}\left(x_{1}, x_{2}\right)=\left(\lambda \cdot x_{1}, \sigma \cdot x_{2}\right)
$$

onde $\tilde{h}$ é a função nas novas coordenadas e $0<\lambda<1<\sigma$ são os autovalores de $D h(p)$. Então, se $g$ é a mudanças de condenadas, $\tilde{h}=g \circ h \circ g^{-1}$. Porém, nas próximas seções omitiremos o sinal $\sim$ de $h$ por simplicidade de notação.

Se $p$ é um ponto fixo de sela de $h$, os conjuntos

$$
\mathcal{W}^{s}(p)=\left\{x \in \mathbb{R}^{2}: \lim _{n \rightarrow \infty} h^{n}(x) \rightarrow p\right\}
$$

$\mathrm{e}$

$$
\mathcal{W}^{u}(p)=\left\{x \in \mathbb{R}^{2}: \lim _{n \rightarrow-\infty} h^{n}(x) \rightarrow p\right\}
$$

são chamados de variedade estável e variedade instável do ponto $p$, respectivamente. Se $p$ é um ponto periódico de período $n$ para $h$, então $p$ é um ponto fixo para $h^{n}$. Logo $\mathcal{W}^{s}(p)$ e $\mathcal{W}^{u}(p)$ serão as variedades estáveis e instáveis com respeito a $h^{n}$. Pelo Teorema da variedade estável, estas variedades são curvas conexas imersas injetivamente que se intersectam transversalmente no ponto $p$, ver [MePa77].

Os ramos estáveis (resp. instáveis) de um ponto periódico de sela $p$ são as duas componentes de $\mathcal{W}^{s}(p) \backslash\{p\}$ (resp. $\left.\mathcal{W}^{u}(p) \backslash\{p\}\right)$. Uma conexão de selas é um ramo $\Gamma$ que e um ramo estável de um ponto periódico de sela $p$ e um ramo instável de um ponto periódico 
de sela $q$, (talvez igual a $p$ ). Um ponto homoclínico de $p$ é um ponto $x \in \mathcal{W}^{s}(p) \cap \mathcal{W}^{u}(p)$. Logo, $h^{n}(x) \rightarrow p$ quando $n \rightarrow \pm \infty$ e é chamado ponto homoclínico transversal quando $\mathcal{W}^{s}(p)$ intersecta $\mathcal{W}^{u}(p) C^{1}$-transversalmente em $x$, ou seja, $T_{x} \mathcal{W}^{s}(p) \oplus T_{x} \mathcal{W}^{u}(p)=T_{x} M$.

Para $\varepsilon>0$ pequeno o suficiente e $p$ um ponto fixo, as variedades estável e instável locais de $h$ no ponto $p$ são, respectivamente,

$$
\begin{aligned}
& \mathcal{W}_{\varepsilon}^{s}(p)=\left\{x \in \mathbb{R}^{2} \mid \text { para todo } n \in \mathbb{N}, d\left(h^{n}(x), p\right) \leq \varepsilon\right\} \mathrm{e} \\
& \mathcal{W}_{\varepsilon}^{u}(p)=\left\{x \in \mathbb{R}^{2} \mid \text { para todo } n \in \mathbb{N}, d\left(h^{-n}(x), p\right) \leq \varepsilon\right\} .
\end{aligned}
$$

Sejam $B^{s}$ uma bola contida em $\mathcal{W}_{\varepsilon}^{s}(p), B^{u}$ uma bola contida em $\mathcal{W}_{\varepsilon}^{u}(p)$ e $V=B^{s} \times B^{u}$. Considere $q \in \mathcal{W}_{\varepsilon}^{s}(p)$ e um disco $D^{u}$ de dimensão $1, C^{1}$-transversal a $\mathcal{W}_{\varepsilon}^{s}(p)$ em $q$. A seguir, enunciaremos um resultado muito útil a respeito de variedades estável e instável, cuja demonstração pode ser encontrada no livro [MePa77].

Teorema 2.1.2. (A-Lema ou Lema da Inclinação). Seja p um ponto fixo hiperbólico para um difeomorfismo $h: \mathbb{R}^{2} \rightarrow \mathbb{R}^{2}$ de classe $C^{k}$. Sejam $\varepsilon>0, V=B^{s} \times B^{u}$ e $q \in \mathcal{W}^{s}(p) \backslash\{p\}$ como acima. Seja $D^{u}$ um disco mergulhado de dimensão 1 tal que $D^{u}$ é $C^{1}$-transversal a $\mathcal{W}_{\varepsilon}^{s}(p)$. Defina $D_{1}^{u}=h\left(D^{u}\right) \cap\left(E^{u}(\varepsilon) \times E^{s}(\varepsilon)\right)$ e $D_{n+1}^{u}=h\left(D_{n}^{u}\right) \cap\left(E^{u}(\varepsilon) \times E^{s}(\varepsilon)\right)$. Então, $D_{n}^{u}$ converge para $\mathcal{W}_{\varepsilon}^{u}(p)$ na topologia $C^{k}$.

Considere $h: \mathbb{R}^{2} \rightarrow \mathbb{R}^{2}$ um homeomorfismo e $m$ a medida de Lebesgue em $\mathbb{R}^{2}$ que associa um número positivo para cada conjunto aberto e não vazio. Nós dizemos que $h$ preserva $m$ quando $m(h(E))=m(E)$ para todo Boreliano $E \subset \mathbb{R}^{2}$. Se $m(h(E))<m(E)$ para todo Boreliano, dizemos que $h$ contrai área. Nos referimos a $m(E)$ como a área do conjunto $E$.

Denotamos o conjunto dos difeomorfismos de classe $C^{r}$ sobre $\mathbb{R}^{2}$ por $\operatorname{Diff} f^{r}\left(\mathbb{R}^{2}\right)$. Uma propriedade para os difeomorfismos de classe $C^{r}$ em $\mathbb{R}^{2}$ é dita ser genérica, se existe um conjunto residual $R \subset \operatorname{Diff} f^{r}\left(\mathbb{R}^{2}\right)$ tal que a propriedade é válida para todo $g \in R$, onde um conjunto de um espaço de Baire é chamado residual caso contenha uma intersecção enumerável de abertos densos. 
No conjuntos do difeomorfismos que preservam áreas em uma superfície compacta, os seguintes são alguns exemplos de propriedades genéricas conhecidas:

G1 Para quaisquer dois pontos periódicos hiperbólicos $p$ e $q$, as intersecções da variedade estável $\mathcal{W}^{s}(p)$ e variedade instável $\mathcal{W}^{u}(q)$ são transversais. Isto foi provado por Robinson [Ro70].

G2 Todos os pontos periódicos são elípticos ou hiperbólicos. Isto também foi provado por Robinson em [Ro70]. G1 e G2 juntos são frequentemente referidos como a condição de Kupka-Smale para difeomorfismos que preservam área.

G3 Para todo ponto periódico hiperbólico $p$, se $\Gamma_{1}$ e $\Gamma_{2}$ são dois ramos da variedade estável ou variedade instável de $p$, então $\overline{\Gamma_{1}}=\overline{\Gamma_{2}}$. Para $S^{2}$, isto foi provado por Mather [Ma82].

G4 Em $S^{2}$, seja $\Gamma_{1}$ um ramo da variedade estável de um ponto periódico hiperbólico $p$ e $\Gamma_{2}$ um ramo da variedade instável de $p$, então $\Gamma_{1} \cap \Gamma_{2} \neq \emptyset$. Isto foi provado por Pixton em [Pi82]. Para toros, este mesmo resultado foi provado por Oliveira em [Oli87].

G5 No conjunto dos difeomorfismo de classe $C^{r}$ que preservam área em $S^{2}$, a união das variedades estável e instável de pontos periódicos hiperbólicos são densas em $S^{2}$. Este resultado foi provado por Franks e Le Calvez [FC03].

O próximo resultado foi provado por Pixton [Pi82]. Em particular, este resultado mostrará, nas nossas hipóteses, que genericamente, se $p$ é um ponto fixo de sela e o número de rotação de fins primos é irracional, então a variedade $\mathcal{W}^{u}(p)$ intersecta transversalmente os dois ramos da variedade estável de todos os pontos periódicos de sela $q \in \overline{\mathcal{W}^{u}(p)}$.

Teorema 2.1.3. Para todo $r \geq 1$, existe um conjunto residual $G_{r} \in \operatorname{Diff}^{r}\left(\mathbb{R}^{2}\right)$ tal que, para todo $g \in G_{r}$ a seguinte propriedade é satisfeita:

Sejam p e q são pontos periódicos de sela. Então, o fecho de um ramo instável de $\mathcal{W}^{u}(p)$ intersecta um ramo estável de $\mathcal{W}^{s}(q)$ se, e somente se, este ramo de $\mathcal{W}^{u}(p)$ intersecta tal ramo de $\mathcal{W}^{s}(q)$ de forma $C^{1}$-transversa. 


\subsection{Uma Propriedade dos continuos}

Os próximos resultados, referentes aos continuos, são de extrema importância para os capítulos posteriores.

Ao longo desta seção, utilizaremos o seguinte resultado clássico, que pode ser encontrado no livro de Topologia Algébrica de Spanier [Spa66]:

Teorema 2.2.1 (Teorema da Curva de Jordan). Seja $J \subset \mathbb{R}^{2}$ um círculo topológico. Então o complementar de $J$ em $\mathbb{R}^{2}$ é a união de dois abertos conexos por caminhos que possuem J como fronteira comum. Além disto, apenas um destes abertos é limitado.

Teorema 2.2.2 (Teorema de Schonflies). Se $\alpha \subset \mathbb{R}^{2}$ é um arco simples, então existe homeomorfismo $g: \mathbb{R}^{2} \rightarrow \mathbb{R}^{2}$ que preserva orientação, tal que $g(\alpha)=[0,1] \times\{0\}$. Se $\gamma \subset \mathbb{R}^{2}$ é uma curva de Jordan, então existe homeomorfismo $g: \mathbb{R}^{2} \rightarrow \mathbb{R}^{2}$ que preserva orientação, tal que $h(\gamma)=\left\{z \in \mathbb{R}^{2}:\|z\|=1\right\}$.

Lema 2.2.1. Seja $\Sigma$ um continuo do plano, cujo complementar é conexo. Se $\alpha$ é um crosscut, então $(\Sigma \cup \alpha)^{c}$ possui componente conexa limitada.

Demonstração. Como $\Sigma \cup \alpha$ é um continuo, existe $r>0$ de modo que $(\Sigma \cup \alpha) \subset B_{r}(0)$. Então, como $B_{r}(0)^{c}$ é um conexo, qualquer outra componente conexa de $(\Sigma \cup \alpha)^{c}$ será limitada e estará contida em $B_{r}(0)$. Para concluir a demonstração do Lema, mostraremos que $(\Sigma \cup \alpha)^{c}$ possui uma componente conexa contida em $B_{r}(0)$. Para isto, iremos supor, por absurdo, que $(\Sigma \cup \alpha)^{c}$ é conexo.

Seja $p \in \alpha \operatorname{com} p \notin \Sigma$. Pelo Teorema de Shonflies, existe um homeomorfismo $g: \mathbb{R}^{2} \rightarrow \mathbb{R}^{2}$ tal que $g(\alpha)=[0,1] \times\{0\}$. Pela escolha de $p$, observe que $g(p)$ não é um extremo de $[0,1] \times\{0\}$. Logo, podemos tomar $\varepsilon_{0}$ suficientemente pequeno de modo que $B_{\varepsilon_{0}}(g(p)) \backslash g(\alpha)$ possui duas componentes conexas e $g(\Sigma) \cap B_{\varepsilon_{0}}(g(p))=\emptyset$. Sejam $A$ e $B$ estas componentes. Tome $a \in A$ e $b \in B$, de modo que o seguimento $\overline{a b}$ intersecta $g(\alpha)$ transversalmente no ponto $p$.

Então, $V=g^{-1}\left(B_{\varepsilon_{0}}(g(p))\right)$ é um aberto, $V \backslash \alpha$ possui duas componentes conexas e 
$V \cap \Sigma=\emptyset$. Além disso, definindo por $\beta=g^{-1}(\overline{a b})$ com extremos nos pontos $x=g^{-1}(a) \mathrm{e}$ $y=g^{-1}(b)$, resulta que $x$ e $y$ estão em diferentes componentes conexas de $V$ e $\beta \cap \alpha=p$.

Agora, note que $(\Sigma \cup \alpha \cup \beta)^{c}$ é conexo, isto porque $(\Sigma \cup \alpha)^{c}$ é conexo e $(\Sigma \cup \alpha) \cap \beta=p$. Então, como conjuntos abertos e conexos de $\mathbb{R}^{n}$ são conexos por caminhos, existe um caminho $\gamma \subset(\Sigma \cup \alpha \cup \beta)^{c}$ com extremos nos pontos $x$ e $y$. Logo, $\gamma \cup \beta$ é uma curva de Jordan e, portanto, segue do Teorema 2.2.1 que $(\gamma \cup \beta)^{c}$ possui exatamente duas componentes conexas. Denote por $U_{1}$ e $U_{2}$ estas componentes.

Como $\alpha \cap(\beta \cup \gamma)=p$, resulta que os pontos finais do crosccut $\alpha$ estão em diferentes componentes conexas de $(\gamma \cup \beta)^{c}$. Consequentemente, $\Sigma \cap U_{1} \neq \emptyset$ e $\Sigma \cap U_{2} \neq \emptyset$. No entanto, como $\Sigma$ é conexo e $\beta \cap \Sigma=\emptyset$, obtemos que $\Sigma \cap \gamma \neq \emptyset$. Mas isto contradiz a hipótese de que $\gamma \subset(\Sigma \cup \alpha)^{c}$. Portanto, $(\Sigma \cup \alpha)^{c}$ possui componente conexa limitada.

Lema 2.2.2. Nas hipóteses do Lema 2.2.1, temos que $(\Sigma \cup \alpha)^{c}$ possui exatamente uma componente conexa limitada.

Demonstração. Inicialmente, provaremos que se $G$ é uma componente conexa de $(\Sigma \cup \alpha)^{c}$, então $\alpha \backslash \Sigma \subset \partial G$. Para isto, dado $p \in \alpha \backslash \Sigma$, utilizando o Teorema de Schonflies como na demonstração do Lema 2.2.1, considere $V_{p}$ uma vizinhança aberta de $p$ tal que $V_{p} \backslash \alpha$ possui exatamente duas componentes conexas.

Fixe $q \in G$ e tome $\varepsilon>0$ satisfazendo $B_{\varepsilon}(p) \subset V_{p}$ e $q \notin B_{\varepsilon}(p)$. Também, considere $S$ uma circunferência de centro em $p$ e raio $r<\varepsilon$. Iniciando no ponto $p$ e percorrendo $\alpha$ nos duas direções possíveis, sejam $A$ e $B$ os primeiros pontos da intersecção $\alpha \cap S$. Como $\Sigma$ é conexo, note que

$$
M=(\Sigma \cup \alpha) \backslash\left(\alpha \cap B_{r}(p)\right)
$$

é compacto, conexo e $M^{c}$ é um conjunto conexo aberto. Isto vai seguir do fato de que $\alpha$ intersecta $\Sigma$ apenas nos dois extremos de $\alpha$ e tomamos $A \neq B$.

Então, considere uma curva $\gamma:[0,1] \rightarrow \mathbb{R}^{2} \backslash M$ tal que $\gamma(0)=p$ e $\gamma(1)=q$. Denotando por $t_{0}=\sup \{t \in[0,1]: \gamma(t) \in S\}$ e $m_{0}=\gamma\left(t_{0}\right)$, observe que 
(i) $t_{0} \neq 1$, pois, caso contrário, $m_{0}=\gamma(1)=q \in S$, uma contradição visto que $S \subset B_{\varepsilon}(p)$ e tomamos $q \notin B_{\varepsilon}(p)$.

(ii) $m_{0} \notin(\Sigma \cup \alpha)$, pois, caso contrário, $m_{0} \in(\alpha \cap S) \subset M$, é isto contradiz o fato de que $\alpha \cap M=\emptyset$.

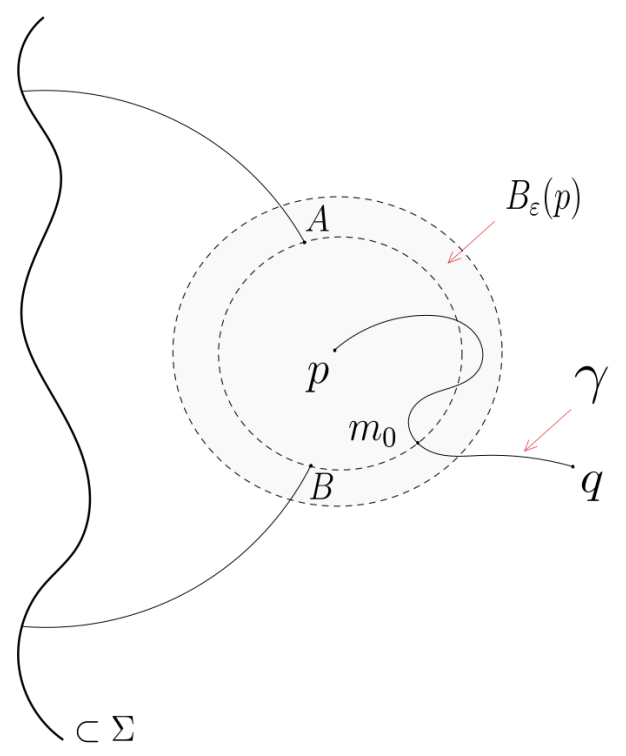

Figura 2.1: Informações dos conjuntos $\Sigma$ e $M$.

Façamos a restrição

$$
\left.\gamma\right|_{\left[t_{0}, 1\right]}:\left[t_{0}, 1\right] \rightarrow \mathbb{R}^{2} \backslash M
$$

Dessa forma, $\gamma\left(\left[t_{0}, 1\right]\right) \subset(\Sigma \cup \alpha)^{c}$, pois se $\gamma\left(\left[t_{0}, 1\right]\right) \cap(\Sigma \cup \alpha) \neq \emptyset$, existiria $t_{1} \in\left(t_{0}, 1\right)$ satisfazendo $\gamma\left(t_{1}\right) \in(\Sigma \cup \alpha) \backslash M$ e, portanto, $\gamma\left(t_{1}\right)$ pertenceria a bola $B_{r}(p)$ que tem $S$ como fronteira. Consequentemente, existiria $t_{2}>t_{1}$ tal que $\gamma\left(t_{2}\right) \in S$, o que se traduz em um absurdo com a definição de $t_{0}$.

Assim, $\gamma\left(\left[t_{0}, 1\right]\right)$ é um caminho inteiramente contido em $(\Sigma \cup \alpha)^{c}$, estando portanto contido em uma mesma componente conexa $G$. Portanto, $m_{0} \in B_{\varepsilon}(p) \cap G$, ficando provado que $B_{\varepsilon}(p) \cap G \neq \emptyset$ para todo $\varepsilon>0$. Então, fazendo $\varepsilon$ convergir para 0 , obtemos que $p \in \partial G$, como queríamos demonstrar. 
Para finalizar a demonstração do Lema, tome $p \in \alpha \backslash \Sigma$. Dado $B_{\varepsilon}(p) \subset V_{p}$, sejam $U_{1}$ e $U_{2}$ as componentes conexas de $B_{\varepsilon}(p) \backslash(\Sigma \cup \alpha)$. Pelo Lema 2.2.1, sabemos que $(\Sigma \cup \alpha)^{c}$ possui pelo menos duas componentes conexas. Sejam $G_{1}$ e $G_{2}$ duas dessas componentes e suponhamos que $U_{1} \subset G_{1}$ e $U_{2} \subset G_{2}$. Supondo por absurdo que exista uma outra componente conexa $G$ de $(\Sigma \cup \alpha)^{c}$, segue da primeira parte desta demonstração que $p \in \partial G, \log \mathrm{o}$, devemos ter

$$
B_{\varepsilon}(p) \cap G \neq \emptyset
$$

Portanto, $G=G_{1}$ ou $G=G_{2}$, ou seja, $(\Sigma \cup \alpha)^{c}$ possui apenas duas componentes conexas.

\subsection{Número de Rotação de Homeomorfismos do Círculo}

Nesta seção iremos tratar de um conceito de extrema importância à teoria subsequente, que é o conceito de número de rotação para homeomorfismos em $S^{1}=\mathbb{R} \backslash \mathbb{Z}$ que preservam orientação. Tal conceito foi apresentado primeiramente por Poincaré em [Po52]. As propriedades dinâmicas dos homeomorfismos do círculo estão intimamente ligadas ao seu número de rotação, conforme descrito por um Teorema de classificação que será apresentado abaixo.

O número de rotação mede o deslocamento médio de pontos no recobrimento. Tal deslocamento médio é feito considerando-se partes finitas da órbita de um determinado ponto no recobrimento e depois tomando-se o limite. Este número independe do ponto $x \in S^{1}$ e as propriedades dos homeomorfismos variam de acordo com sua racionalidade ou não.

Definição 2.3.1. Seja $h: S^{1} \rightarrow S^{1}$ um homeomorfismo que preserva orientação. Um homeomorfismo $H: \mathbb{R}^{2} \rightarrow \mathbb{R}^{2}$ é um levantamento de $h$ se

$$
h(\pi(\tilde{x}))=\pi(H(\tilde{x}))
$$


$\operatorname{com} \pi: \mathbb{R} \rightarrow S^{1}$ a aplicação de recobrimento definida por $\pi(\tilde{x})=\tilde{x} \bmod 1$.

Se $H$ é um levantamento de $h$, não é difícil verificar que um homeomorfismo $G: \mathbb{R} \rightarrow \mathbb{R}$ também é um levantamento de $h$ se, e somente se, $G(\tilde{x})=H(\tilde{x})+n$, para algum $n \in \mathbb{Z}$. Isto porque $H-G$ é uma função contínua e assumindo valores inteiros, logo constante.

Proposição 2.3.1 (Poincaré). Seja $h: S^{1} \rightarrow S^{1}$ um homeomorfismo que preserva orientação e $H: \mathbb{R} \rightarrow \mathbb{R}$ um levantamento de $h$ para o recobrimento universal. Então o limite

$$
\rho(H)=\lim _{n \rightarrow \infty} \frac{H^{n}(\tilde{x})-\tilde{x}}{n}
$$

existe para todo $\tilde{x} \in \mathbb{R}$, é independente de $\tilde{x}$ e está definido a menos de um inteiro, isto é, se $H$ e $G$ forem levantamentos de $h$, então $\tau(H)-\tau(G) \in \mathbb{Z}$.

Definição 2.3.2. Seja $h: S^{1} \rightarrow S^{1}$ um homeomorfismo que preserva orientação $e$ $H: \mathbb{R} \rightarrow \mathbb{R}$ um levantamento de $h$. O número

$$
\rho(h)=\pi(\rho(H))
$$

diz-se o número de rotação de $h$, com $H$ qualquer levantamento de $h$ e $\pi: \mathbb{R} \rightarrow S^{1}$ a aplicação de recobrimento.

A Proposição 2.3.1 garante que, para todo homeomorfismo do círculo que preserva orientação, o número $\rho(h)$ existe, está bem definido e independe de $x$.

Outro resultado relevante é a seguinte Proposição que afirma que o número de rotação é um invariante topológico da dinâmica.

Proposição 2.3.2. Sejam $h$ e g homeomorfismos do círculo que preservam orientação. Se $h$ e g são topologicamente conjugados, então $\rho(h)=\rho(g)$.

A próxima proposição mostra como o conceito de número de rotação serve de critério para garantir a existência de pontos periódicos para um homeomorfismo $h: S^{1} \rightarrow S^{1}$ que preserva orientação. 
Proposição 2.3.3 (Poincaré). Seja $h: S^{1} \rightarrow S^{1}$ um homeomorfismo que preserva orientação.

- $\rho\left(h^{n}\right)=n \rho(h)$ para todo $n \in \mathbb{Z}$.

- $\rho(h) \in \mathbb{Q}$ se, e somente se, $h$ tem um ponto periódico e todas as órbitas periódicas têm o mesmo período, que é igual ao denominador de $\rho(h)$, pensando na fração irredutivel.

Exemplo 2.3.1. Entre os tipos mais simples de homeomorfismos de $S^{1}$ que preservam orientação podemos citar as rotações. Seja $\alpha \in[0,1)$ e considere a rotação $R_{\alpha}$ em $S^{1}$. Lembremos que a órbita de um ponto $x \in S^{1}$ pela rotação $R_{\alpha}$ é

$$
O_{R_{\alpha}}(x)=\{x+n \alpha \quad(\bmod 1): n \geq 0\} .
$$

Considere um levantamento $\tilde{R}_{\alpha}(x)=x+\alpha+k$. Se $\alpha$ é um número racional, $\alpha=p / q$ com $p$ e q primos entre si, então $q \alpha=p \in \mathbb{Z}$, e neste caso a órbita $O_{R_{\alpha}}(x)$ é periódica com período q. Quando a é irracional, não existem órbitas periódicas e a órbita de qualquer ponto é densa em $S^{1}$. Em ambos os casos,

$$
\begin{aligned}
\rho\left(R_{\alpha}\right) & =\lim _{n \rightarrow \infty} \frac{\tilde{R}_{\alpha}^{n}(x)-x}{n} \quad(\bmod 1) \\
& =\lim _{n \rightarrow \infty} \frac{x+\alpha n+k n-x}{n}(\bmod 1) \\
& =\alpha+k(\bmod 1) \\
& =\alpha .
\end{aligned}
$$

O seguinte Teorema remonta ao trabalho de Poincaré e Denjoy [De32] e classifica o comportamento dinâmico dos homeomorfismos do círculo que preservam orientação em termos do número de rotação.

Teorema 2.3.1 (Classificação dos homeomorfismos do círculo). Seja $h: S^{1} \rightarrow S^{1} u m$ homeomorfismo que preserva orientação.

- Assuma que $\rho(h)=p / q$, com $p$ e q primos entre si. Então, $h$ tem pelo menos uma órbita com período q e toda órbita periódica deve ter o mesmo período q. Adicionalmente, toda órbita positiva ou negativa converge para uma órbita periódica. 
- Assuma que $\rho(h) \notin \mathbb{Q}$. Existem duas possibilidades:

- Se existir uma órbita densa, então h é topologicamente conjugada a uma rotação irracional. Portanto toda órbita é densa.

- Caso contrário, existe um conjunto invariante de Cantor $C$ que é o único conjunto minimal de $S^{1}$ tal que, cada órbita positiva e negativa converge para $C$.

O homeomorfismo que pertence a este último tipo, possuindo número de rotação irracional e um conjunto invariante de Cantor, é chamado de homeomorfismo de Denjoy. Uma propriedade muito importante para tais homeomorfismos é que eles não podem existir em classe $C^{2}$, ver [De32].

Dados dois pontos $x$ e $y$ em $S^{1} \operatorname{com} x \neq y$, veja que existem exatamente dois intervalos em $S^{1}$ com extremos em $x$ e $y$. Se um número de rotação $\rho(h)$ é irracional, então todo ponto $x \in S^{1}$ não é periódico e podemos considerar um intervalo $I$ como um dos intervalos com extremos nos pontos $x$ e $h(x)$. O próximo Lema, que será importante no Capítulo 4 , verifica-se para qualquer escolha do intervalo $I$ com extremos em $x$ e $h(x)$.

Lema 2.3.1. Considere $h: S^{1} \rightarrow S^{1}$ um homeomorfismo que preserva orientação, $x \in S^{1}$ e $I \subset S^{1}$ um intervalo fechado com extremos nos pontos $x$ e $h(x)$. Se o número de rotação $\rho(h)$ for irracional, temos que a semi-órbita positiva ou negativa de qualquer ponto y em $S^{1}$ intersecta $I$.

Demonstração. Vamos provar o Lema para semi-órbitas positivas, visto que a demonstração para para semi-órbitas negativas é exatamente análoga. Considere $\left\{h^{n}(y)\right\}_{n \in \mathbb{N}}$ a semi-órbita positiva de $y$. Para provarmos o lema, veja que é suficiente mostrarmos que os iterados para trás de $I$ cobrem $S^{1}$, ou seja, que

$$
S^{1} \subset \bigcup_{k \in \mathbb{N}} h^{-k}(I)
$$

Para isto, defina $I_{k}=h^{-k}(I)$ e note que a sequência $\left\{I_{k}\right\}_{k \in \mathbb{N}}$ é formada por intervalos consecutivos, ou seja, dado $k \in \mathbb{N}$, os intervalos $I_{k}$ e $I_{k+1}$ têm o ponto final $h^{-k}(y)$ em 
comum. Logo, se $S^{1} \neq \bigcup_{k \in \mathbb{N}} I_{k}$ então a sequência de pontos $\left\{h^{-k}(y)\right\}_{k \in \mathbb{N}}$, que são os extremos dos intervalos $I_{k}$, converge para algum $z \in S^{1}$. Se isto acontecer, veja que $z$ é um ponto fixo, pois

$$
z=\lim _{k \rightarrow \infty} h^{-k}(y)=\lim _{k \rightarrow \infty} h^{-k+1}(y)=h\left(\lim _{k \rightarrow \infty} h^{-k}(y)\right)=h(z)
$$

Como isto contradiz a hipótese de que $\rho(h)$ é irracional, o lema está provado.

\subsection{Teoria de Fins Primos}

Seja $h: \mathbb{R}^{2} \rightarrow \mathbb{R}^{2}$ um homeomorfismo e $U \subset \mathbb{R}^{2}$ um disco topológico aberto tal que $h(U)=U$. Se $\partial U$ é uma curva de Jordan, então $\partial U$ é homeomorfo a $S^{1}$ e podemos associar um número de rotação $\rho(h, U)$ como o número de rotação de $\left.h\right|_{\partial U}$. Neste caso, a teoria desenvolvida por Poincaré nos diz que existe ponto periódico em $\partial U$ se, e somente se, o número de rotação $\rho(h, U)$ é racional. No entanto, em geral, este resultado de Poincaré não é válido quando $\partial U$ não é uma curva de Jordan.

A teoria de fins primos, desenvolvida por Carathéodory [Ca13] em 1913, nos permite substituir a fronteira de um domínio conexo e simplesmente conexo por uma fronteira "ideal" com a propriedade de que, com a topologia que surge naturalmente da sua construção, esta nova fronteira é homeomorfa ao círculo. Uma breve descrição da teoria de fins primos será apresentada a seguir. Para mais informações e provas, o leitor pode consultar [Ma82, Po91].

Dado um homeomorfismo $h$ do plano ou da esfera que preserva orientação, considere $U$ um aberto conexo e simplesmente conexo invariante por $h$. Um sequência de crosscuts $\left\{c_{n}\right\}_{n \in \mathbb{N}}$ é dita uma cadeia de crosscuts se satisfaz as seguintes condições:

- $c_{n}$ separa $c_{n-1}$ e $c_{n+1}$, isto é, $c_{n-1}$ e $c_{n+1}$ estão em diferentes componentes de $U \backslash c_{n}$.

- $\bar{c}_{n} \cap \bar{c}_{n+1}=\emptyset$ para todo $n \in \mathbb{N}$.

Associada a uma cadeia $\left\{c_{n}\right\}_{n \in \mathbb{N}}$ de crosscuts, existe a sequência $\left\{V_{n}\right\}_{n \in \mathbb{N}}$ tal que $V_{n}$ é a 
componente de $U \backslash c_{n}$ que contém $c_{n+1}$. Uma sequência $V_{1} \supset V_{2} \supset V_{3} \supset \ldots$ é chamada de cadeia.

Dizemos que uma cadeia $\left\{V_{n}\right\}_{n \geq 0}$ divide outra cadeia $\left\{V_{n}^{\prime}\right\}_{n \geq 0}$ quando, dado qualquer inteiro $n$, existir um $m$ de modo que $V_{m} \subset V_{n}^{\prime}$. Duas cadeias que se dividem mutualmente são chamadas de cadeias equivalentes. É fácil verificar que isto define um relação de equivalência. Uma cadeia $V=\left\{V_{n}\right\}_{n \geq 0}$ é chamada de cadeia prima se qualquer cadeia que dividir $V$ for equivalente a $V$. Um fim primo de $U$ é a classe de equivalência de uma cadeia prima. O conjunto dos fins primos de $U$ será denotado por $\mathbb{P}=\mathbb{P}(U)$.

Para definirmos uma topologia em $U^{*}=U \cup \mathbb{P}$, considere $\mathfrak{p} \in U^{*}$ um fim primo e assuma que a cadeia $\left\{V_{1} \supset V_{2} \supset \ldots\right\}$ representa $\mathfrak{p}$. Dado $W$ um subconjunto aberto de $U$, diremos que o fim primo $\mathfrak{p}$ divide $W$ se existir um $i$ tal que $V_{i} \subset W$. Denote por

$$
\tilde{W}=\{\mathfrak{p} \in \mathbb{P}(U): \mathfrak{p} \text { divide } W\}
$$

o conjunto de todos os fins primos que dividem $W$. Definimos uma base de abertos para a compactificação de fins primos de $U$ como a família de todos os conjuntos $\tilde{W}$, com $W$ um aberto em $U$, juntamente com o conjunto de todos os abertos de $U$. Estes abertos formam uma base para nossa topologia natural em $U^{*}$.

Com esta topologia, uma sequência $\left\{\mathfrak{p}_{n}\right\}_{n \in \mathbb{N}}$ de fins primos converge para um fim primo $\mathfrak{p}$ representado pela cadeia $\left\{V_{n}\right\}_{n \in \mathbb{N}}$ quando, para todo $m$, existe um $N(m) \in \mathbb{N}$ tal que $\mathfrak{p}_{n} \in \tilde{V}_{m}$ para todo $n>N(m)$. O conjunto $U^{*}$ com esta topologia é chamado de compactificação de fins primos de $U$.

O próximo Teorema, devido à Carathéodory, é um clássico e fundamental resultado da teoria de fins primos e nos diz que $U^{*}$ é topologicamente o disco unitário fechado. O enunciado abaixo foi retirado de um artigo de Cartwright e Littlewood sobre existência de pontos fixos em um continuo do plano [Ca13]:

Teorema 2.4.1. (Carathéodory) Seja $U \subset S^{2}$ um aberto conexo e simplesmente conexo. Suponha que que $\partial U$ possui mais de um ponto, e que $\mathbb{D}=\left\{p \in \mathbb{R}^{2}:\|p\| \leq 1\right\}$. Então existe um homeomorfismo $\tau: U^{*} \rightarrow \mathbb{D}$, de maneira que os pontos em $U$ são enviados em 
pontos no interior do disco $\mathbb{D}$, e os fins primos de $U^{*}$ correspondem a pontos da fronteira $S^{1}$. Além disto, se h é um homeomorfismo de $S^{2}$ que deixa $U$ invariante, então existe uma aplicação $\tilde{h}$ em $U^{*}$, de maneira que $\tilde{h}=h$ em $U$.

Com este Teorema, conseguimos estudar a dinâmica de $h$ na fronteira de $U$ estudando a correspondente dinâmica em $S^{1}$. Como fins primos são mandados em fins primos por $h$, e a fronteira de $U$ é $h$-invariante, temos que $h$ induz um homeomorfismo $\tilde{h}$ em $U^{*}$. Seja $\tau$ o homeomorfismo entre $U^{*}$ e $\bar{D}$. Então o círculo $S^{1}$ é invariante pelo homeomorfismo $h^{\prime}=\tau \circ \tilde{h} \circ \tau^{-1}$ de $\bar{D}$ que preserva orientação, visto que $h$ preserva orientação. Este fato é consequência de resultados conhecidos da teoria de variedades com bordo. Portanto, podemos estudar a dinâmica de $h$ na fronteira de $U$ através do homeomorfismo $h^{\prime}$, associando um número de rotação para $h$ e $U$ definido por $\rho(h, U):=\rho\left(\left.h^{\prime}\right|_{S^{1}}\right)$.

Definição 2.4.1. Considere $h: S^{2} \rightarrow S^{2}$ um homeomorfismo que preserva orientação e que deixa invariante um conjunto aberto $U$ conexo e simplesmente conexo. Seja $h^{\prime}: \bar{D} \rightarrow \bar{D}$ o homeomorfismo induzido por $h$, como definido acima. O número de rotação de fins primos de $h$ em $U$ é definido por

$$
\rho(h, U):=\rho\left(\left.h^{\prime}\right|_{S^{1}}\right)
$$

Observação 2.4.1. Seja $\gamma \subset U$ um crosscut com pontos finais nos pontos $a, b$ e denote por $V$ uma cross-section de $U \backslash \gamma$. Então, o conjunto $\tilde{V}$ é um intervalo aberto em $\mathbb{P}(U)$. O fecho de $\tilde{V}$ é um intervalo fechado cujos pontos finais são pontos acessíveis a e $\tilde{b}$, determinados pelo crosscut $\gamma$.

Tendo em vista estas definições, é uma questão particularmente relevante saber em que medida a teoria de Poincaré para os homeomorfismos do círculo que preservam orientação se estende a este novo número invariante: o que se pode dizer sobre a dinâmica de $h$ na fronteira de $U$, conhecendo o número de rotação de fins primos de $h$ em $U$ ?

Ao contrário do caso clássico do círculo, exemplos mostram que é possível ter $\rho(h, U)=$ 0 mas não existir ponto fixo em $\partial U$. Também é possível que o número de rotação $\rho(h, U)$ seja irracional e exista ponto fixo na fonteira de $U$. De um modo geral, para obter algum 
tipo de conexão entre as duas noções, é preciso fazer hipóteses adicionais ao homeomorfismo ou na região $U$. O próximo Teorema, que é importante para os próximos capítulos, tornase um resultado particularmente útil por não exigir tais hipóteses adicionais. Para uma demonstração, ver [KLN15].

Teorema 2.4.2. Seja $h: \mathbb{R}^{2} \rightarrow \mathbb{R}^{2}$ um homeomorfismo que preserva orientação e $U \subsetneq \mathbb{R}^{2}$ um aberto conexo, simplesmente conexo e invariante por h. Se existir um ponto fixo acessivel em $\partial U$, então $\rho(h, U)=0$.

Considere $\left\{V_{n}\right\}_{n \in \mathbb{N}}$ uma cadeia que representa um fim primo p. Como cada $V_{n}$ é conexo, temos que a intersecção $\bigcap_{n \geq 1} \overline{V_{n}}$ é um subconjunto conexo e compacto de $S^{2}$. Então, tal intersecção ou é um único ponto ou um continuo. Chamamos

$$
I(\mathfrak{p})=\bigcap_{n=1}^{\infty} \overline{V_{n}}
$$

de impressão do fim primo $\mathfrak{p}$. Veja que a definição de impressão não depende da cadeia que representa o fim primo $\mathfrak{p}$, pois se $\left\{V_{n}\right\}_{n \in \mathbb{N}}$ divide $\left\{V_{n}^{\prime}\right\}_{n \in \mathbb{N}}$, então $I\left(V_{n}\right) \subset I\left(V_{n}^{\prime}\right)$, o mesmo acontece para o oposto. Desse modo, a impressão de um fim primo está bem definida. Observe que dois fins primos diferentes podem ter a mesma impressão.

Dado um fim primo $\mathfrak{p}$, um ponto $p$ em $I(\mathfrak{p})$ é chamado de ponto principal de $\mathfrak{p}$ se existir uma sequência $\left\{c_{n}\right\}_{n \in \mathbb{N}}$ de crosscuts que definem $\mathfrak{p}$ e de modo que o $n$-ésimo crosscut $c_{n}$ converge para $p$, isto é, para qualquer vizinhança de $p$, existe $n$ tal que o crosscut $c_{n}$ está contido nesta vizinhança. O conjunto destes pontos é chamado de conjunto principal de $\mathfrak{p}$ e será denotado por $\Pi(\mathfrak{p})$.

Os próximos lemas aparecem, por exemplo, em [Ma82] (como o Teorema 17.1 e Corolário 15 , respectivamente).

Lema 2.4.1. O conjunto principal de um fim primo $\mathfrak{p}$ tem um único ponto $p$ se, e somente se, $p$ é acessível.

Lema 2.4.2. O conjunto principal de um fim primo é um continuo.

Vamos introduzir a seguinte notação: se $\left(K_{i}\right)_{i \in \mathbb{N}}$ é uma sequência de conjuntos, então $K_{i} \rightarrow x \in S^{2}$ quando $i \rightarrow \infty$ se, para cada vizinhança aberta $V$ de $x$, existe $i_{0}$ tal que 
$K_{i} \subset V$ para todo $i \geq i_{0}$. O próximo resultado está contido em [Mat82], Lemas 3.1-3.4 e Proposição 3.7. Este resultado mostrará, em particular, que associado a cada fim primo, existe uma cadeia $\left\{c_{n}\right\}_{n \geq 0}$ de crosscuts que define este fim primo, tal que $\operatorname{diam}_{S^{2}}\left(c_{n}\right) \rightarrow 0$ quando $n \rightarrow \infty$.

Proposição 2.4.1. As seguintes propriedades acontecem:

1. Uma cadeia $\left\{V_{n}\right\}_{n \geq 0}$ de $U$ é prima se, e somente se, existe um $x \in \partial U$ e alguma cadeia equivalente $\left\{V_{i}^{\prime}\right\}_{i \in \mathbb{N}}$ tal que $\left.\partial V_{i}^{\prime}\right|_{U} \rightarrow x$ quando $i \rightarrow \infty$.

2. Se $\lambda:[0,1) \rightarrow U$ é um endcut, então existe um fim primo $\mathfrak{p} \in U^{*}$ tal que $\lambda(t) \rightarrow \mathfrak{p}$ em $U^{*}$ quando $t \rightarrow 1^{-}$.

Isto nos mostra que pontos acessíveis e fins primos estão intimamente relacionados. Além disso, é possível definir fins primos usando apenas estes tipo de cadeias. Com o próxima Lema, fins primos acessíveis formam um conjunto denso em $\mathbb{P}(U)$.

Lema 2.4.3. Suponha que $\partial U$ é um conjunto compacto. Então, o conjunto dos pontos acessiveis de $\partial U$ por $U$ é um conjunto denso em $\partial U$.

Demonstração. Tomemos um ponto $x \in \partial U$ e fixe um $\varepsilon>0$. Como $x \in \partial U$, existe $y \in B_{\varepsilon}(x) \cap U$. Seja $\gamma:[0,1] \rightarrow \mathbb{R}^{2}$ uma parametrização do segmento de reta com extremos em $x$ e $y$, de modo que $l(0)=y$. Seja $t_{0}=\min \{t \geq 0: l(t) \in \partial U\}$. Como $l(0) \in U$ e $l(1) \in \partial U$, temos que $t_{0} \in(0,1]$ e $l\left(\left[0, t_{0}\right)\right) \subset U$. Portanto, $l\left(\left[0, t_{0}\right)\right) \subset U$ é um endcut acessando o ponto $l\left(t_{0}\right)$. Logo, arbitrariamente próximo de $x$, existem pontos acessíveis.

Observação 2.4.2. Supondo que tanto $U$ quando $\partial U$ sejam h-invariantes, se um endcut $\gamma$ acessa um ponto $p \in \partial U$, temos que $h(\gamma)$ é um endcut acessando $h(p) \in \partial U$. Desta forma, o Lema 2.4.3 nos permite concluir que o conjunto de pontos acessíveis de $\partial U$ é um subconjunto h-invariante e denso em $\partial U$.

O próximo Teorema apresenta uma descrição da dinâmica do conjunto de pontos acessíveis para um difeomorfismo $h$ do plano ou da esfera, com as hipóteses adicionais de 
que todos os pontos periódicos na fronteira de $U$ são hiperbólicos, onde $U$ é um aberto contendo um ponto fixo atrator $x$ e, para todo $y \in U, \lim h^{n}(y)=x$ quando $n \rightarrow \infty$. Para um demonstração, ver Teorema 6.1 em [AY92].

Teorema 2.4.3. Assuma que os pontos periódicos de $h \in \partial U$ são hiperbólicos, onde $h e$ $U$ são como acima. Se o número de rotação $\rho(h, U)$ é racional, então todo ponto acessível ou é um ponto periódico ou está na variedade estável de um ponto periódico acessível. 


\section{Capítulo 3}

\section{Alguns Resultados 1}

Neste capítulo, apresentaremos propriedades da classe de homeomorfismos $\mathcal{H}$, objeto de estudo deste trabalho e algumas particularidades dinâmicas no plano, gerado pela iteração de um homeomorfismo $h \in \mathcal{H}$ que satisfaz duas propriedades locais que estão ligadas pela dinâmica. Também iremos apresentar os primeiros resultados obtidos nesta tese, a saber, o Teorema 3.1.1 e o Teorema 3.1.4.

\subsection{Sobre à Classe de Homeomorfismos $\mathcal{H}$}

Seja $h$ um homeomorfismo de $\mathbb{R}^{2}$. Um ponto fixo $a$ de $h$ é chamado de atrator se existir um disco aberto e limitado $V$ contendo $a$ tal que

$$
h(\bar{V}) \subset V \quad \text { e } \bigcap_{i \in \mathbb{N}} h^{i}(V)=\{a\}
$$

Para este $V$, o conjunto

$$
U_{a}=\bigcup_{i \in \mathbb{N}} h^{-i}(V)
$$

é chamado de bacia de atração de a. A bacia de atração de um ponto fixo atrator é um subconjunto aberto, invariante, conexo e simplesmente conexo. Além disso, considerando 
a extensão de $h$ para $S^{2}$, quando $U_{a}$ é um subespaço próprio de $S^{2}$ e a fronteira de $U$ não se reduz a um ponto, a teoria de fins primos de Carathéodory pode ser aplicada. Um ponto $x \in U_{a}$ tem a propriedade de que

$$
\lim _{n \rightarrow \infty} h^{n}(x) \rightarrow a
$$

Um ponto fixo atrator $b$ para o homeomorfismo inverso $h^{-1}$ é chamado de repulsor para $h$ e a bacia $U_{b}$ de atração de $b$ por $h^{-1}$, também chamada de bacia de repulsão de $h$, é definida de modo análogo. Estas bacias, de atração e repulsão de pontos fixos, como já falamos, são conjuntos abertos, invariantes por $h$ e homeomorfas a discos abertos de $\mathbb{R}^{2}$.

O conjunto $\omega$-limite de um ponto $p \in \mathbb{R}^{2}$, denotado por $\omega(p)$, é definido como o conjunto (possivelmente vazio) dos pontos $x \in \mathbb{R}^{2}$ para os quais existe uma sequência de inteiros $\left\{n_{k}\right\}_{k \geq 1}$ convergindo para $+\infty$, tal que

$$
\lim _{k \rightarrow \infty} h^{n_{k}}(p) \rightarrow x
$$

Dizemos que a origem $0 \in \mathbb{R}^{2}$ é um atrator global para $h$ quando for um atrator local e $\omega(p)=\{0\}$, para todo $p \in \mathbb{R}^{2}$.

Finalmente, chamamos um conjunto $\Sigma \subset \mathbb{R}^{2}$ de atrator global para $h$ quando, para alguma vizinhança compacta $K \subset \mathbb{R}^{2}$ de $\Sigma$, tenhamos:

(a) Para todo $p \in \mathbb{R}^{2}$, existe $n>0$ tal que $h^{n}(p) \in K$.

(b) $h(K) \subset \operatorname{int}(K)$, e

(c) $\bigcap_{n=1}^{\infty} h^{n}(K)=\Sigma$.

Dado $h \in \mathcal{H}$, como o infinito é um repulsor para $h$, considere $D$ o disco aberto limitado por uma curva de Jordan $\gamma$, suficientemente grande e que enlaça a origem, tal que $h(\gamma) \subset D$. Estaremos interessados em estudar a dinâmica de $h$ no conjunto:

$$
\Sigma=\bigcap_{n=0}^{\infty} h^{n}(D) .
$$




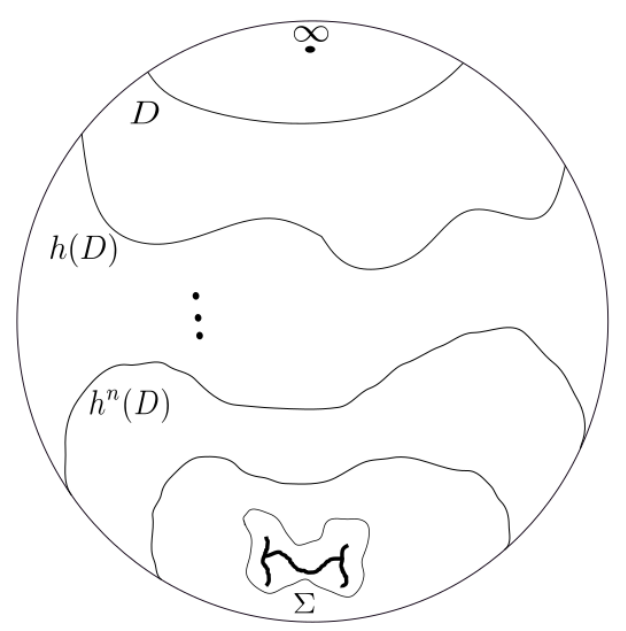

Figura 3.1: O conjunto $\Sigma$ visto como um subconjunto da esfera.

As propriedades importantes do atrator $\Sigma$ estão presentes na próxima proposição, para uma demonstração, ver [Go07].

Proposição 3.1.1. Dado $h \in \mathcal{H}$ e $U$ a bacia de repulsão do infinito, temos que o atrator $\Sigma=\partial U$ é compacto, conexo, h-invariante e como hé dissipativo, ele tem interior vazio. Mais ainda, $S^{2}=\Sigma \cup U$.

A definição de dissipatividade de $h \in \mathcal{H}$ nos diz que, para todo aberto $D \subset \mathbb{R}^{2}$, a área $h^{n}(D) \rightarrow 0$, quando $n \rightarrow \infty$. Caso $h$ seja um difeomorfismo, esta é uma condição mais fraca do que exigir que $\operatorname{det}\left(D h_{x}\right)<1$ para todo ponto. Nas diferentes hipóteses, resultados diferentes valem ou ainda não se sabe, por exemplo, não se sabe existe um difeomorfismo $h \in \mathcal{H} \operatorname{com} \operatorname{det}\left(D h_{x}\right)<1$ para todo ponto nas condições do Teorema 3.1.4.

Um homeomorfismo $h \in \mathcal{H}$ tem a propriedade importante de que a medida de Lebesgue de $\Sigma$ é zero. De fato, dado $A$ um aberto que contém $\Sigma$, como $\Sigma$ é invariante e a medida de Lebesque de $h^{n}(A)$ converge para zero quando $n \rightarrow \infty$, obtemos que $m(\Sigma)=0$. Além disso, a bacia $U_{\infty}$ tem medida infinita.

Observação 3.1.1. Tando $\Sigma$ quando o seu complementar em $\mathbb{R}^{2}$ são h-invariantes, portanto, segue da Observação 2.4.2 que o conjunto de pontos acessíveis de $\Sigma$ é um subconjunto h-invariante e denso em $\Sigma$. 
Dados $h \in \mathcal{H}$ e $U=U_{\infty}$ a bacia de repulsão do infinito, estaremos interessados em estudar a relação do número de rotação $\rho(h, U)$ com a existência de pontos periódicos acessíveis. Para o caso em que $\rho(h, U)$ é racional, provamos o seguinte teorema:

Teorema 3.1.1. Para todo $h \in \mathcal{H}$ com $\rho(h, U)$ racional, existe ponto periódico diferente da origem em $\partial U$. Adicionalmente, supondo que $h$ é um difeomorfismo de classe $C^{2} e$ que $\operatorname{det}\left(\left.D h\right|_{x}\right)<1$ para todo $x \in \mathbb{R}^{2}$, então este ponto periódico é acessível.

A demonstração deste Teorema será apresentada na seção 3.2. A primeira parte dele apareceu em [OR11] com uma demonstração diferente.

Em vista do Teorema 3.1.1, naturalmente surge a seguinte pergunta para os casos em que o número de rotação $\rho(h, U)$ é irracional:

Pergunta 3.1.1. Se $h \in \mathcal{H}$ e o número de rotação $\rho(h, U)$ é irracional, então a origem é o único ponto periódico de $h$ ?

Em [COR10], Luiz Corbato, Rafael Ortega e Ruiz del Portal apresentaram exemplos que motivam a pergunta anterior. Neste mesmo artigo, adicionando a hipótese de que a origem é acessível pela bacia de repulsão do infinito, $U$, os autores apresentaram uma resposta parcial positiva.

Teorema 3.1.2. Seja $h \in \mathcal{H}$ e suponha que a origem é acessível pela bacia de repulsão do infinito, $U$. Então, as seguinte afirmações são equivalentes:

- O número de rotação $\rho(h, U)$ é irracional.

- O único ponto periódico de h é a origem.

Em [Mat13], Matsumoto mostrou que a condição de acessibilidade da origem no Teorema 3.1.2 é necessária para que a origem seja o único ponto periódico de $h$.

Teorema 3.1.3. Para quaisquer números reais $\alpha$ e $\beta$, existe um homeomorfismo $h \in \mathcal{H}$ tal que $\rho\left(h, U_{\infty}\right)=\alpha$ e $\rho\left(h, U_{0}\right)=\beta$, onde $U_{\infty}$ é a bacia de repulsão de $\infty$ e $U_{0}$ a bacia de atração de 0 por $h$. 
Com este Teorema, a condição de acessibilidade da origem torna-se necessária, uma vez que, podemos escolher $\alpha$ irracional e $\beta$ racional e, pelo Teorema 3.1.1, existirá ponto periódico para $h$ na fronteira de $U_{\infty}$. Desse modo, apenas a hipótese de irracionalidade no número de rotação de fins primos da compactificação de fins primos de $U_{\infty}$ não é suficiente para garantir que a origem é o único ponto periódico de $h$. Além disso, este Teorema mostra que a existência de pontos periódicos na fronteira de um aberto $U$ conexo, simplesmente conexo e invariante por $h$, não implica que $\rho(h, U)$ é racional.

Em vista do resultado de Matsumoto, a Pergunta 3.1.1 é reformulada para:

Pergunta 3.1.2. Considere $h \in \mathcal{H}$ e $U$ a bacia de repulsão do infinito. Se o número de rotação $\rho(h, U)$ for irracional, é possivel existir um ponto periódico de sela com intersecção homoclínica na fronteira de $U$ ?

Usando um exemplo de Addas Zanata e Veiga Gomes em [BS11] e as ideias do trabalho de Matsumoto [Mat13], é possível construir um exemplo de um homeomorfismo $h \in \mathcal{H}$ que demonstra o Teorema a seguir.

Teorema 3.1.4. Para todo $\alpha \in(0,1)$ irracional, existe $h \in \mathcal{H}$ com número de rotação $\rho(h, U)=\alpha$ e tal que $\partial U$ possui um ponto $p$ de sela com intersecção homoclínica.

Nas próximas seções, apresentaremos resumidamente o exemplo de Matsumoto e o exemplo de Addas Zanata com Veiga Gomes. Utilizaremos estes exemplos para construir um homeomorfismo $h$ nas condições do Teorema 3.1.4.

\subsection{Demonstração do Teorema 3.1.1}

Se $h$ é um homeomorfismo do círculo que preserva orientação e tem um ponto periódico de período $n$, como já dissemos, pelo Teorema da classificação dos homeomorfismos do círculo, é conhecido que todo ponto do círculo ou é um ponto fixo por $h^{n}$ ou sua órbita converge para um ponto de período $n$ sob iterados de $h^{n}$. 
No seguinte conjunto de definições, descreveremos noções de estabilidade para pontos periódicos. Frequentemente, mencionamos apenas pontos fixos, mas considerando que um ponto periódico de período $n$ é um ponto fixo para $h^{n}$, as definições e resultados que seguem são satisfeitas para pontos periódicos.

Considere $h$ um homeomorfismo do círculo que preserva orientação. Um ponto fixo $p$ em $S^{1}$ é chamado:

(1) Atrator por um lado, se existe uma vizinhança em um dos lados de $p$ tal que todos os pontos convergem para $p$ por iterados de $h$.

(2) Repulsor por um lado, se existe uma vizinhança em um dos lados de $p$ tal que todos os pontos convergem para $p$ por iterados de $h^{-1}$.

Com esta definição, um ponto fixo isolado $p \in S^{1}$ é um atrator ou um repulsor em cada um de seus lados. Definições análogas são feitas para o espaço de fins primos e a palavra "ponto"é substituída pelo termo "fim primo". Se $p$ é um ponto fixo atrator (resp. repulsor) por um do lado, então pelo Teorema de Carathéodory, o fim primo associado $\mathfrak{p}$ será atrator (resp. repulsor) por um lado.

Agora, suponha que $h$ é um homeomorfismo do plano, $U$ é um disco topológico aberto e $\tilde{h}$ é o homeomorfismo no conjunto $U^{*}$, induzido por $h$. Apesar de que fins primos fixos correspondem a pontos fixos em $S^{1}$, não é verdade que um fim primo fixo por $\tilde{h}$ necessariamente contém um ponto que é fixo por $h$. Nos próximos Lemas, considere que $h \in \mathcal{H}$. Estes Lemas são importantes porque relacionam pontos fixos de $h$ em $\partial U=\Sigma$ com fins primos fixos de $\tilde{h}$ em $\mathbb{P}(U)$. Para uma demonstração do Lema 3.2.1, ver [CL51].

Lema 3.2.1. Seja $\mathfrak{p}$ um fim primo de $U^{*}$ fixo por $\tilde{h}$ e seja $\left\{c_{n}\right\}_{n \in \mathbb{N}}$ uma sequência de crosscuts convergindo para um ponto principal $p$ de $\mathfrak{p}$. Se, para todo $n$, o crosscut $h\left(c_{n}\right)$ tem pelo menos um ponto em comum com $c_{n}$, então $p$ é um ponto fixo de $h$.

Lema 3.2.2. Se $\Sigma=\partial U$ onde $U$ é a bacia de atração de um ponto fixo e $\mathfrak{p}$ é um fim primo fixo que atrai por pelo menos um lado, então todo ponto principal de $\mathfrak{p}$ é um ponto fixo de $h$. 
Demonstração. Considere $\mathfrak{p}$ um fim primo de $\mathbb{P}(U)$ que atrai por um lado e considere $p$ um ponto principal do fim primo $\mathfrak{p}$. Por definição de ponto principal, existe uma sequência de crosscuts $\left\{c_{n}\right\}_{n \in \mathbb{N}}$ que define uma cadeia $V=\left\{V_{n}\right\}_{n \in \mathbb{N}}$ com

$$
V_{n} \subset\left(\Sigma \cup c_{n}\right)^{c} \text { e } c_{n+1} \subset V_{n}
$$

de modo que $c_{n} \rightarrow p$ quando $n \rightarrow \infty$.

Como $\mathfrak{p}$ atrai por pelo menos um lado, mostraremos que não pode existir uma subsequência $\left\{m_{n}\right\}_{n \in \mathbb{N}}$ de modo que $h\left(c_{m_{n}}\right) \cap c_{m_{n}}=\emptyset$ para todo $n$. Consequentemente, descartando uma quantidade finita de termos da sequência $\left\{c_{n}\right\}_{n \in \mathbb{N}}$, podemos assumir que $h\left(c_{n}\right) \cap c_{n} \neq \emptyset$ para todo $n$. Então, usando o Lema 3.2.1, concluiremos que $p$ é um ponto fixo.

Dado o crosscut $c_{i}$, como os pontos finais de $c_{i}$ são pontos acessíveis, denotemos por $a_{i}$ e $b_{i}$ os pontos finais do crosscut $c_{i}$ e por $\tilde{a}_{i}$ e $\tilde{b}_{i}$ os fins primos acessíveis associados aos pontos $a_{i}$ e $b_{i}$, respectivamente.

Como estamos supondo que o fim primo $\mathfrak{p}$ atrai por pelo menos um lado por $\tilde{h}$, denote por $I$ o lado de $\mathfrak{p}$ tal que, se um fim primo $\mathfrak{p}_{0} \in I$, então

$$
\lim \tilde{h}^{m}\left(\mathfrak{p}_{0}\right)=\mathfrak{p} \text { quando } m \rightarrow \infty
$$

Desse modo, jogando fora alguns termos da sequência $\left\{c_{n}\right\}_{n \in \mathbb{N}}$ se necessário, podemos assumir que para todo $n$, no círculo de fins primos $S^{1}$, um extremo de $c_{n}$ pertence a $I$. Seja $\tilde{a}_{n}$ este extremo. Portanto

$$
\lim _{m \rightarrow \infty} \tilde{h}^{m}\left(\tilde{a}_{n}\right)=\mathfrak{p}
$$

onde $\tilde{h}^{m}\left(\tilde{a}_{n}\right) \in I$ é um fim primo que é um extremo do crusscut $\tilde{h}^{m}\left(c_{n}\right)$.

Suponhamos agora que nesta sequência de crosscuts exista um crosscut $c_{n}$ satisfazendo $h\left(c_{n}\right) \cap c_{n}=\emptyset$. Então, como $\mathfrak{p}$ atrai por um lado, a existência de tal crosscut $c_{n}$ implica que, no círculo de fins primos, $\tilde{h}\left(\overline{V_{n}}\right) \subset V_{n}$. Consequentemente, $\tilde{h}^{m}\left(\overline{V_{n}}\right) \subset V_{n}$ para todo $m \in \mathbb{N}$, ver figura 3.2. Por este motivo, concluí-se que, em $\mathbb{R}^{2}$, 


$$
h^{m}\left(\overline{V_{n}}\right) \subset V_{n} \text { para todo } m \in \mathbb{N} \text {. }
$$

Mas note que este fato contradiz a hipótese de que $U$ é a bacia de atração de um ponto fixo em $\mathbb{R}^{2} \backslash \Sigma$.

A seguir, apresentaremos a prova do Teorema 3.1.1.

Demonstração. Considere que $\rho(h, U)=p / q$, com $p$ e $q$ primos entre si. Para facilitar a notação, vamos assumir que $q=1$ (para o caso geral, basta substituir $h$ por $h^{q}$ ). Inicialmente, assuma o caso em que o homeomorfismo $\tilde{h}$ no círculo de fins primos $\mathbb{P}(U)$ seja a identidade. Provemos a seguinte afirmação:

Afirmação 1. Suponha que $\left.\tilde{h}\right|_{\mathbb{P}(U)}$ é a identidade. Então, todos os pontos acessíveis de $h$ em $\Sigma$ são fixos por $h$.

De fato, se $x \in \Sigma$ é um ponto acessível e $\gamma:[0,1) \rightarrow U$ um endcut acessando $x$, então a Proposição 2.4.1 nos diz que existe um fim primo $\mathfrak{p} \in \mathbb{P}(U)$ tal que $\gamma(t) \rightarrow \mathfrak{p}$ em $U^{*}$ quando $t \rightarrow 1^{-}$. Como o fim primo $\mathfrak{p}$ é fixo por $\tilde{h}$, note que $h(\gamma(t)) \rightarrow \mathfrak{p}$ em $U^{*}$ quando $t \rightarrow 1^{-}$. Desse modo, temos que $h(x)=x$ pois, caso contrário,

$$
\lim h(\gamma(t))=\tilde{h}(\mathfrak{p}) \neq \mathfrak{p}=\lim \gamma(t)
$$

em $U^{*}$, quando $t \rightarrow 1^{-}$. Logo, se $\left.\tilde{h}\right|_{\mathbb{P}(U)}$ é a identidade, todo ponto acessível em $\Sigma$ é um ponto fixo por $h$.
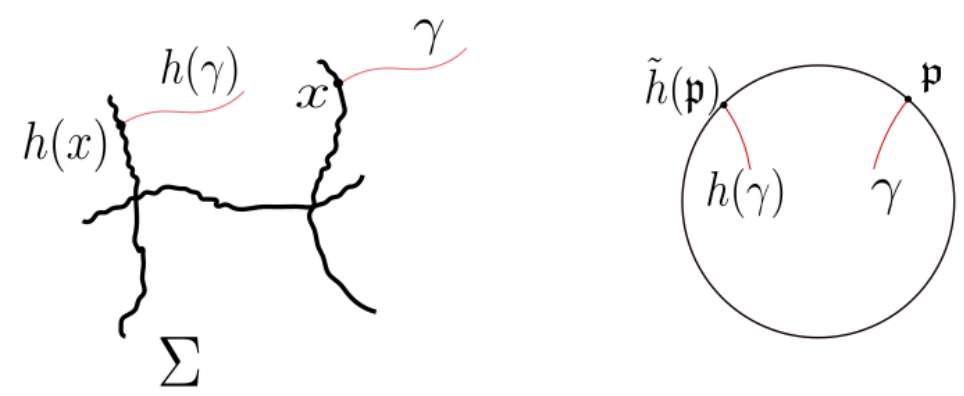

Figura 3.2: Se $x \neq h(x)$ em $\mathbb{R}^{2}$, então $\mathfrak{p} \neq \tilde{h}(\mathfrak{p})$ em $\mathbb{P}(U)$. 
Vamos assumir então que o homeomorfismo $\left.\tilde{h}\right|_{\mathbb{P}(U)}$ não é a identidade. Inicialmente, iremos provar que existe um ponto fixo para $h$ que é diferente da origem.

Como $\rho\left(h^{-1}, U\right)=-\rho(h, U)=0$ e $\left.\tilde{h}\right|_{\mathbb{P}(U)}$ não é a identidade, existe um fim primo fixo $\mathfrak{p} \in \mathbb{P}(U)$ que atrai por pelo menos um lado por iterados de $h^{-1}$. Tome $p \in \Pi(\mathfrak{p})$ um ponto principal do fim primo p. Por definição, existe uma sequência de crosscuts $\left\{c_{n}\right\}_{n \in \mathbb{N}}$, que define uma cadeia $V=\left\{V_{n}\right\}_{n \in \mathbb{N}}$ com

$$
V_{n} \subset\left(\Sigma \cup c_{n}\right)^{c} \text { e } c_{n+1} \subset V_{n}
$$

de modo que $c_{n} \rightarrow p$ quando $n \rightarrow \infty$. O Lema 3.2.2 mostra que $p$ é um ponto fixo.

Com a finalidade de provar (por absurdo) a existência de um outro ponto fixo para $h$ em $\Sigma$ além da origem, podemos assumir que a origem é o único ponto periódico de $h$, ou seja, vamos supor que $p=0$.

Sendo 0 um ponto fixo atrator de $h$, considere $U_{0}$ a bacia de atração de 0 por $h$. Logo, por definição, existe disco aberto $A \subset U$ satisfazendo

$$
h(\bar{A}) \subset A \text { e } 0=\bigcap_{n=1}^{\infty} h^{n}(\bar{A}) .
$$

Observe que, como a sequência de crosscuts $\left\{c_{n}\right\}_{n \in \mathbb{N}}$ converge para 0 quando $n \rightarrow \infty$, podemos descartar uma quantidade finita de elementos desta sequência e assumir que $c_{n} \subset A$ para todo $n \in \mathbb{N}$. Em particular, isto implica que

$$
\lim _{m \rightarrow \infty} h^{m}\left(c_{n}\right)=0
$$

Além disso, para todo elemento $c_{i}$ desta sequência de crosscuts $\left\{c_{n}\right\}_{n \in \mathbb{N}}$, pelo menos um dos seguintes casos acontece:

(i) Existe subsequência infinita $m_{n}^{\prime} \in \mathbb{N}$, de modo que

$$
h^{m_{n}^{\prime}}\left(c_{i}\right) \cap c_{i}=\emptyset \text {, para todo } n \in \mathbb{N} \text {. }
$$

(ii) Existe subsequência infinita $m_{n}^{\prime \prime} \in \mathbb{N}$, de modo que 


$$
h^{m_{n}^{\prime \prime}}\left(c_{i}\right) \cap c_{i} \neq \emptyset \text {, para todo } n \in \mathbb{N} \text {. }
$$

No entanto, mostraremos que a hipótese de 0 ser o único ponto fixo de $h$ implica que, para todo crosscut $c_{i}$ da sequência $\left\{c_{n}\right\}_{n \in \mathbb{N}}$, os dois casos $(i)$ e $(i i)$ não podem ocorrer. Mas veja que isto é um absurdo, visto que não é possível que cada um dos casos ocorra apenas para uma quantidade finita de inteiros. Logo, concluiremos que a origem não pode ser o único ponto periódico de $h$, implicando que o ponto periódico $p \neq 0$.

Afirmação 2. Para todo crosscut $c_{i}$ em $\left\{c_{n}\right\}_{n \in \mathbb{N}}$, não existe subsequência $\left\{m_{n}^{\prime}\right\}_{n \in \mathbb{N}}$ satisfazendo o caso $(i)$.

Para demonstrar esta afirmação, vamos supor por absurdo que existe um crosscut $c_{i}$ e uma subsequência $\left\{m_{n}^{\prime}\right\}_{n \in \mathbb{N}}$ satisfazendo $h^{m_{n}^{\prime}}\left(c_{i}\right) \cap c_{i}=\emptyset$. Para facilitar a notação, vamos denotar por $\{m\}_{m \in \mathbb{N}}$ tal subsequência, isto é, $h^{m}\left(c_{i}\right) \cap c_{i}=\emptyset$ para todo $m \in \mathbb{N}$. Consequentemente, o mesmo acontece no conjunto de fins primos $\mathbb{P}(\mathbb{U})$, ou seja, para todo $m \in \mathbb{N}$,

$$
\tilde{h}^{m}\left(c_{i}\right) \cap c_{i}=\emptyset, \text { em } U^{*} \simeq \bar{D}
$$

Visto que os pontos finais de um crosscut são pontos acessíveis, denotemos por $a_{i}$ e $b_{i}$ os pontos finais do crosscut $c_{i}$ e por $\tilde{a}_{i}$ e $\tilde{b}_{i}$ os fins primos acessíveis associados aos pontos $a_{i}$ e $b_{i}$, respectivamente.

Novamente, como $\lim _{n \rightarrow \infty} c_{n}=0$, descartando alguns termos da sequência $\left\{c_{n}\right\}_{n \in \mathbb{N}}$ se necessário, podemos assumir que para todo $i$, no círculo de fins primos $S^{1}$ um extremo de $c_{i}$ pertence a $I$, intervalo atrator para $\tilde{h}$ lateral a $\mathfrak{p}$. Seja $\tilde{a}_{i}$ este extremo. Então

$$
\lim _{m \rightarrow \infty} \tilde{h}^{-m}\left(\tilde{a}_{i}\right)=\mathfrak{p}
$$

onde $\tilde{h}^{-m}\left(\tilde{a}_{i}\right) \in I$ é um fim primo que é um extremo do crusscut $\tilde{h}^{-m}\left(c_{i}\right)$.

Como $\tilde{h}^{-m}\left(c_{i}\right) \cap c_{i}=\emptyset$, então $\tilde{h}^{-m}\left(c_{i}\right) \subset \tilde{V}_{i}$ para todo $m \in \mathbb{N}$, ver figura 3.2. Por este motivo, concluí-se que, em $\mathbb{R}^{2}$, 


$$
h^{-m}\left(c_{i}\right) \subset V_{i} \text { para todo } m \in \mathbb{N} \text {. }
$$

Logo, isto mostra que, tomando $x \in h^{-m}\left(c_{i}\right)$ para algum $m$, como $h^{-r}(x) \in V_{i}$ para todo $r$, temos que $x$ não pode pertencer a $U$, que é a bacia de atração do infinito por $h^{-1}$. Isto completa a demonstração da afirmação 2 .

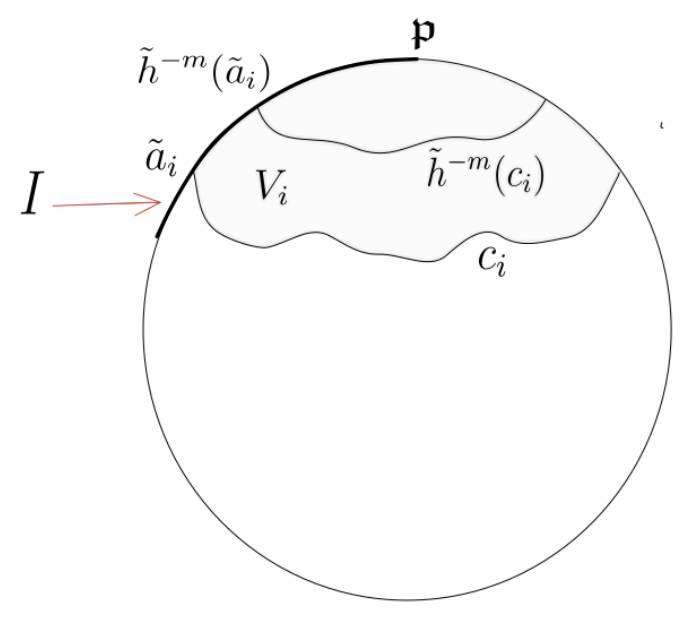

Figura 3.3: Informações na compactificação de fins primos de $U$.

Afirmação 3. Para todo crosscut $c_{i}$ em $\left\{c_{n}\right\}_{n \in \mathbb{N}}$, não existe sequência $\left\{m_{n}^{\prime \prime}\right\}_{n \in \mathbb{N}}$ satisfazendo o caso $(i i)$.

Para a demonstração de tal afirmação, suponhamos por absurdo que existe um crosscut $c_{i}$ e que existe uma sequência $\left\{m_{n}^{\prime \prime}\right\}_{n \in \mathbb{N}}$ que satisfaz $h^{m_{n}^{\prime \prime}}\left(c_{i}\right) \cap c_{i} \neq \emptyset$ para todo $n \in \mathbb{N}$. Novamente, para facilitar a notação, suponhamos que $h^{m}\left(c_{i}\right) \cap c_{i} \neq \emptyset$ para todo $m \in \mathbb{N}$.

Como no caso $(i)$, denote por $a_{i}$ e $b_{i}$ os pontos acessíveis que são os pontos extremais do crosscut $c_{i}$ e denote por $\overline{c_{i}}=c_{i} \cup\left\{a_{i}, b_{i}\right\}$. Sendo $\overline{c_{i}}$ é um compacto, temos que $\mathrm{d}\left(\overline{c_{i}}, 0\right)=\varepsilon$ para algum $\varepsilon>0$. Em consequência, $h^{m}\left(c_{i}\right) \cap c_{i} \neq \emptyset$ para todo $m \in \mathbb{N}$ implica que

$$
\lim _{m \rightarrow \infty} h^{m}\left(c_{i}\right) \neq 0
$$

Porém, isto entra em contradição com a nossa escolha de crosscuts, visto que tomamos uma sequência de crosscuts $\left\{c_{n}\right\}_{n \in \mathbb{N}} \subset A$, que está contido da bacia de atração de 0 por $h$. Esta contradição finaliza a demonstração da afirmação 3. 
Observe que, as contradições nos casos $(i)$ e $(i i)$ surgiram quando assumimos que $\operatorname{Per}(h)=\{0\}$. Portanto, quando $h \in \mathcal{H}$ e o número de rotação $\rho(h, U)=0$, obrigatoriamente, existe ponto fixo para $h$ além da origem.

Para demonstrar a segunda parte do Teorema, na próxima afirmação utilizaremos a hipótese de que $\operatorname{det}\left(\left.D h\right|_{x}\right)<1$ para todo $x \in \mathbb{R}^{2}$ e mostraremos que o conjunto principal do fim primo $\mathfrak{p}$ contém apenas o ponto $p$. Portanto, utilizando o Lema 2.4.1, concluiremos que $p$ é ponto fixo e acessível. Por este motivo, para finalizarmos a demonstração deste Teorema, provemos a seguinte afirmação:

Afirmação 4. Se $h \in D i f f^{2}\left(\mathbb{R}^{2}\right)$ e $\operatorname{det}\left(\left.D h\right|_{x}\right)<1$ para todo $x \in \mathbb{R}^{2}$, então o conjunto principal de $\mathfrak{p}$, contém um único ponto $p$. Portanto, pelo Lema 2.4.1, $p$ é fixo e acessível.

Antes de iniciarmos a prova desta afirmação, precisaremos de alguns resultados que serão apresentados a seguir.

Para uma demonstração do próximo Lema, ver por exemplo Carr [Ca81], Teorema 1, página 16 e Lema 1, página 20, onde o resultado foi provado para campos de vetores, demonstrações análogas valem para difeomorfismos, como indicado nas páginas 33-35.

Lema 3.2.3. Assuma que $h: \mathbb{R}^{2} \rightarrow \mathbb{R}^{2}$ é um difeomorfismo de classe $C^{2}$ que fixa a origem e Dh(0,0) tem 1 e $\lambda$ como autovalores, com $0<\lambda<1$. Se escrevermos $h$ em coordenadas tais que $h(x, y)=(x+u(x, y), \lambda y+v(x, y))$, onde as funções $u, v e$ suas primeiras derivadas tem valor 0 na origem, então existe uma função $g$ de classe $C^{2}$, definida para $|x|$ suficientemente pequeno, tal que $g(0)=g^{\prime}(0)=0$, cujo gráfico é invariante por iterados de $h$, uma vizinhança $U$ de $(0,0)$ e $C>0$ de modo que, para todo segmento de órbita $\left(x_{k}, y_{k}\right)_{0 \leq k \leq n}$ contido em $U$, temos que $\left|y_{n}-g\left(x_{n}\right)\right| \leq C \lambda^{n}\left|y_{0}-g\left(x_{0}\right)\right|$.

Como consequência do Lema anterior, temos interesse no seguinte resultado:

Corolário 3.2.1. Assuma que $h: \mathbb{R}^{2} \rightarrow \mathbb{R}^{2}$ é um difeomorfismo de classe $C^{2}$ que fixa a origem e Dh(0,0) tem 1 e $\lambda$ como autovalores, com $0<\lambda<1$. Considere a função $g$ definida para $|x|$ pequeno, a vizinhança $U$ e a constante $C$ dadas pelo Lema anterior. 
Então, se existir algum ponto fixo $\left(x_{0}, y_{0}\right) \in U$, temos que $y_{0}=g\left(x_{0}\right)$, ou seja, todo ponto fixo $\left(x_{0}, y_{0}\right)$ de $h$ que esta contido em $U$ pertence ao gráfico de $g$.

Demonstração. De fato, supondo que $\left(x_{0}, y_{0}\right) \in U$ é um ponto fixo, observe que a órbita $\left(x_{n}, y_{n}\right)=h^{n}\left(x_{0}, y_{0}\right)=\left(x_{0}, y_{0}\right)$ é fixa. Logo, usando o Lema 3.2.3, obtemos que para todo $n \in \mathbb{N}$,

$$
\left|y_{0}-g\left(x_{0}\right)\right| \leq C \lambda^{n}\left|y_{0}-g\left(x_{0}\right)\right|
$$

Como $\lambda<1$, fazendo $n \rightarrow \infty$, conclui-se que $y_{0}=g\left(x_{0}\right)$. Isto mostra que o ponto $\left(x_{0}, y_{0}\right)$ pertence ao gráfico de $g$.

Demonstração da Afirmação 4 .

A hipótese $\operatorname{det}\left(\left.D h\right|_{x}\right)<1$ para todo $x \in \mathbb{R}^{2}$ nos diz que, para todo $x \in \mathbb{R}^{2}$, pelo menos um autovalor tem módulo menor que 1.

Suponha que os dois autovalores de $\left.D h\right|_{p}$ possuem módulo diferente de um. Então, $p$ é ponto fixo isolado. Logo, como $U$ é bacia de atração do infinito por $h^{-1}$ e o fim primo $\mathfrak{p}$ atrai por pelo menos um lado por $\tilde{h}^{-1}$, o Lema 3.2.2 nos mostra que todos os pontos principais de $\Pi(\mathfrak{p})$ são fixos por $h$. Sendo $p$ um ponto fixo isolado, obtemos que $\Pi(\mathfrak{p})=p$. Consequentemente, pelo Lema 2.4.1, temos que $p$ é um ponto fixo acessível. Então, iremos assumir que $\operatorname{det}\left(\left.D h\right|_{p}\right)$ possui 1 e $\lambda \in(0,1)$ como autovalores.

Por absurdo, suponhamos que o conjunto principal de $\mathfrak{p}$ não se reduz a apenas um ponto. Então, os Lemas 2.4.1 e 2.4.2 garantem que П(p) é um continuo não-degenerado e sem pontos acessíveis. Além disso, o Lema 3.2.2 nos mostra que todo ponto de $\Pi(\mathfrak{p})$ é fixo por $h$. Seja $p$ um ponto fixo de $\Pi(\mathfrak{p})$. Via uma mudança de coordenadas, assumamos que $p$ é a origem. Então, considerando a função $g$ do Corolário 3.2.1, resulta que

$$
\Pi(\mathfrak{p}) \cap U \subset\left\{(x, y) \in \mathbb{R}^{2}: y=g(x)\right\},
$$

ou seja, os pontos fixos de $\Pi(\mathfrak{p}) \cap U$ estão contidos no gráfico de $g$. Portanto, como $\Pi(\mathfrak{p}) \cap U$ é um continuo de pontos fixos (porque seque do Lema 2.4.2 que $\Pi(\mathfrak{p})$ é um 
continuo), a dinâmica de $h$ em uma vizinhança de $\Pi(\mathfrak{p}) \cap U$ é como na figura 3.4.

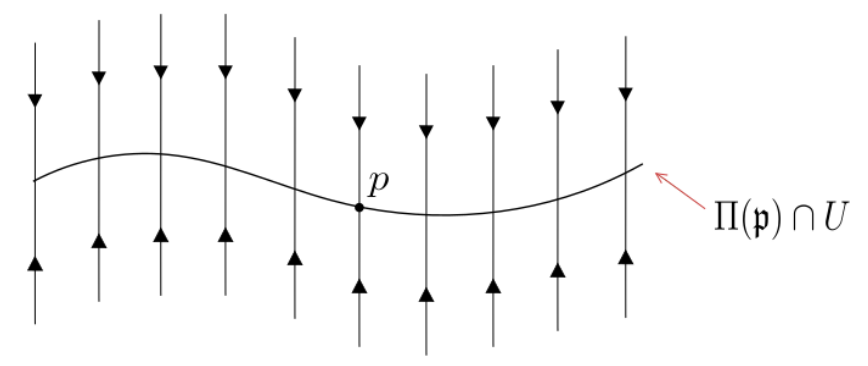

Figura 3.4: A dinâmica de $h$ em uma vizinhança de $\Pi(\mathfrak{p}) \cap U$.

Tomamos o fim primo $\mathfrak{p}$ de modo que este atrai por pelo menos um lado por $\tilde{h}^{-1} \mathrm{em}$ $\mathbb{P}(U)$. Logo $\mathfrak{p}$ repele por um lado por $\tilde{h}$.

Agora, tome $p_{0} \in \Pi(\mathfrak{p}) \cap U$ um ponto principal do fim primo $\mathfrak{p}$ e considere $V \subset U$ uma vizinhança aberta de $p_{0}$, como na figura 3.5. Para todo $x \in V$, observe que

$$
\lim _{m \rightarrow \infty} h^{m}(x) \in \Pi(\mathfrak{p})
$$

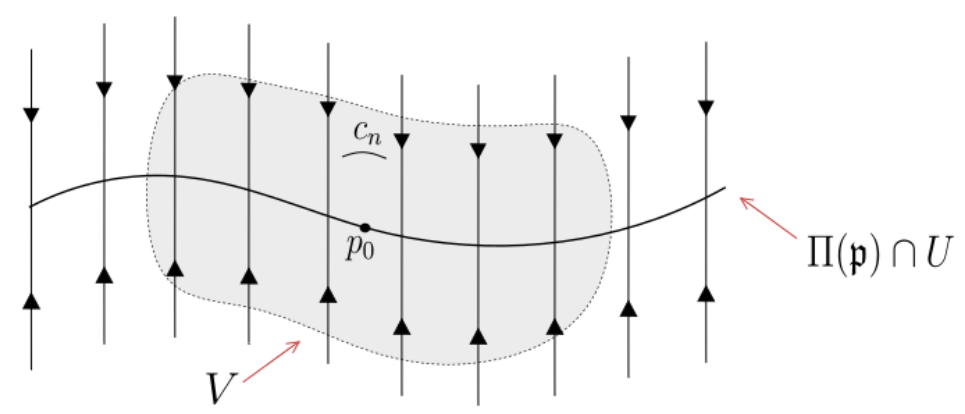

Figura 3.5: A dinâmica de $h$ perto de $\Pi(\mathfrak{p}) \cap U, p_{0} \in \Pi(\mathfrak{p}) \cap U$ e $V$ uma vizinhaça aberta de $p$.

Sendo $p_{0}$ um ponto principal, considere $\left\{c_{n}\right\}_{n \in \mathbb{N}}$ uma sequência de crosscuts satisfazendo $c_{n} \rightarrow p_{0}$ quando $n \rightarrow \infty$. Visto que $V$ é uma vizinhança aberta de $p_{0}$, podemos descartar alguns elementos desta sequência de crosscuts e assumir que $c_{n} \in V$ para todo $n$.

Como $c_{n} \subset U$ e os pontos extremos de cada $c_{n}$ são pontos acessíveis de $\Sigma \backslash \Pi(\mathfrak{p})$, resulta que 


$$
\overline{c_{n}} \cap \Pi(\mathfrak{p})=\emptyset .
$$

Por outro lado, dado que $c_{n} \in V$, quando $m \rightarrow \infty$, temos que,

$$
\mathrm{d}\left(h^{m}\left(\overline{c_{n}}\right), \Pi(\mathfrak{p})\right) \rightarrow 0 .
$$

Consequentemente, existe $m>0$ de modo que

$$
h^{m}\left(\overline{c_{n}}\right) \cap \overline{c_{n}}=\emptyset .
$$

Agora, com este fim primo $\mathfrak{p}$ repele por um lado por $\tilde{h}$ e a sequência de crosscuts $\left\{c_{n}\right\}_{n \in \mathbb{N}}$ converge para $p_{0}$ quando $n \rightarrow \infty$, alguns argumentos da demonstração da Afirmação 2 nos mostram que existe $n_{0} \in \mathbb{N}$ de modo que, se $n \geq n_{0}$, então $h^{m}\left(c_{n}\right) \nsubseteq V_{n}$, onde $V_{n}$ é a componente conexa aberta de $\left(\Sigma \cup c_{n}\right)^{c}$ que contém $c_{n+1}$. Por este motivo, fixando $n>n_{0}$, segue de 3.1 a existência de $m>0$ tal que $h^{m}\left(c_{n}\right) \cap V_{n}=\emptyset$.

No entanto, repetindo os detalhes na demonstração da afirmação 2, obtemos que $h^{-m}\left(c_{n}\right) \subset V_{n}$ para todo $m \in \mathbb{N}$. Novamente, isto contradiz a hipótese de que o infinito é um atrator para $h^{-1}$ em $U=\mathbb{R}^{2} \backslash \Sigma$.

O Teorema 2.4.2 junto com o Teorema 3.1.1 fornecem diretamente o próximo Corolário.

Corolário 3.2.1. Assuma que $h \in \mathcal{H}$ é um difeomorfismo de classe $C^{2}$. Adicionalmente, suponha que $\operatorname{det}\left(\left.D h\right|_{x}\right)<1$ para todo $x \in \mathbb{R}^{2}$. Então o número de rotação $\rho(h, U)$ é racional se, e somente se, existe ponto periódico acessível $p \in \Sigma$.

\subsection{O exemplo de Matsumoto}

Nesta seção, iremos apresentar o homeomorfismo que Matsumoto usou para demonstrar o Teorema 3.1.3. Para isto, considere a esfera menos os dois polos, $S^{2} \backslash\{0, \infty\}$, que é homeomorfa ao cilindro $\mathbb{A}=S^{1} \times \mathbb{R}$. Se 0 e $\infty$ são pontos fixos de um homeomorfismo 
$h: S^{2} \rightarrow S^{2}$, que preserva orientação, então $h$ induz um homeomorfismo que preserva orientação em $\mathbb{A}$, e vice-versa, que também iremos denotar por $h$.

Para demonstrar o Teorema 3.1.3, Matsumoto construiu um homeomorfismo $h \in \mathcal{H}$ e mostrou que podemos tomar $h$ de modo que $\rho\left(h, U_{\infty}\right)=\alpha$ e $\rho\left(h, U_{0}\right)=\beta$ para $\alpha$ e $\beta$ fixados. A seguir, apresentaremos os principais passos da construção de tal homeomorfismo. Inicialmente, considere o cilindro $\mathbb{A}=S^{1} \times \mathbb{R} \cong S^{2} \backslash\{0, \infty\}$.

No subdomínio $S^{1} \times[5, \infty), h$ é da forma

$$
h(\theta, t)=\left(f_{\alpha}(\theta), t-g_{\alpha}(\theta, t)\right),
$$

onde $f_{\alpha}$ é a rotação em $S^{1}$ se $\alpha$ é racional e um homeomorfismo de Denjoy caso contrário, com o número de rotação de $f_{\alpha}$ igual a $\alpha$. Escolhendo uma função $g_{\alpha}$ apropriada, com $g_{\alpha}\left(\left(S^{1} \times[5,+\infty[)) \subset[0,1]\right.\right.$, podemos obter um homeomorfismo $h$ que tem um único conjunto minimal $C_{\alpha}$ em $t=10$ e com $h$ satisfazendo também

$$
h\left(S^{1} \times[5, \infty)\right)=S^{1} \times[4, \infty) .
$$

Do mesmo modo, nós definimos $h$ em $(\infty,-5]$ usando um homeomorfismo $f_{\beta}$ de $S^{1}$ com número de rotação $\beta$ e tal que $h$ tem um único conjunto minimal $C_{\beta}$ em $t=-10$. Finalmente, em $S^{1} \times[-5,5]$, definimos $h$ como

$$
h(\theta, t)=\left(\varphi_{t}(\theta), t-1\right)
$$

usando uma isotopia $\varphi_{t}$ para $t \in[-5,5]$, que cola $f_{\alpha} \operatorname{com} f_{\beta}$.

Para a construção de $h$, uma função contínua $g_{\alpha}: S^{1} \rightarrow S^{1}$ é tomada de maneira que

(a) $g_{\alpha}^{-1}(0)=C_{\alpha}$, e

(b) para todo $\theta \in S^{1} \backslash C_{\alpha}$,

$$
\sum_{i \geq 0} g_{\alpha}\left(f_{\alpha}^{i}(\theta)\right)=\infty \text { e } \sum_{i \leq 0} g_{\alpha}\left(f_{\alpha}^{i}(\theta)\right)=\infty
$$


Após provar a existência de $f_{\alpha}, C_{\alpha}$ e $g_{\alpha}$ (analogamente para $f_{\beta}, C_{\beta}$ e $g_{\beta}$ ) com as condições requeridas, Matsumoto construiu uma função contínua $g_{0}: S^{1} \times \mathbb{R} \rightarrow[0,1]$, diferenciável ao longo de $\mathbb{R}$, e que satisfaz

(c) $g_{0}^{-1}(0)=\left(C_{\alpha} \times\{10\}\right) \cup\left(C_{\beta} \times\{-10\}\right)$,

(d) para $\left.t \in[9,11], g_{0}(\theta, t) \geq g_{\alpha}(\theta)\right)$ e para $\left.t \in[-11,-9], g_{0}(\theta, t) \geq g_{\beta}(\theta)\right)$, com a igualdade apenas quando $t= \pm 10$,

(f) $g_{0}=1 \mathrm{em} S^{1} \times((-\infty, 15] \cup[-5,5] \cup[15, \infty))$, e

(e) $\partial g_{0} / \partial t<1$

Agora, escolha uma família contínua $\varphi_{t}(t \in \mathbb{R})$ de homeomorfismos de $S^{1}$ tal que $\varphi_{t}=f_{\alpha}$ para $t \in[5, \infty)$ e $\varphi_{t}=f_{\beta}$ para $t \in(-\infty,-5]$. Então, Matsumoto mostrou que $h: S^{1} \times \mathbb{R} \rightarrow S^{1} \times \mathbb{R}$, definido por

$$
h(\theta, t)=\left(\varphi_{t}(\theta), t-g_{0}(\theta, t)\right),
$$

é um homeomorfismo que satisfaz as condições do Teorema 3.1.3. Além disso,

$$
U_{\infty} \cap\left(S^{1} \times[5, \infty)\right)=\left(S^{1} \times[5, \infty)\right) \backslash\left(C_{\alpha} \times[5,10]\right)
$$

Para mostrar que $\rho\left(h, U_{\infty}\right)=\alpha$, Matsumoto usou que, para qualquer $\theta \in S^{1}$, o raio $r_{\theta}:(0, \infty) \rightarrow \infty$ definido por

$$
r_{\theta}(t)=\left(\theta, t^{-1}+10\right)
$$

satisfaz a propriedade: para todo $\theta \notin C_{\alpha}$, o ponto extremo de $r_{\theta}(\infty)=(\theta, 10)$ é um ponto em $U_{\infty}$ e para todo $\theta \in C_{\alpha}$, o pronto extremo de $r_{\theta}(\infty)$ é um ponto acessível que corresponde a um fim primo acessível em $S^{1}$, com número de rotação $\alpha$. Desse modo, Matsumoto mostrou que $\rho\left(h, U_{\infty}\right)=\alpha$ e $\rho\left(h, U_{0}\right)=\beta$.

Com a demonstração do Teorema 3.1.3, Matsumoto produziu exemplos de homeomorfismos $h \in \mathcal{H}$ com número de rotação $\rho\left(h, U_{\infty}\right)$ irracional e com órbita periódica em $\partial U_{\infty}$. Logo, a irracionalidade de $\rho\left(h, U_{\infty}\right)$ não caracteriza a não existência de órbitas periódicas na fronteira da bacia de atração do infinito. 


\subsection{O exemplo de Bernado e Salvador}

Em [BS11], Bernado e Salvador construíram um difeomorfismo $h \in \mathcal{H}$ que possui um ponto $p \in \Sigma$ de sela com intersecção homoclínica transversal, fato que implica na existência de infinitos pontos periódicos hiperbólicos. Este difeomorfismo satisfaz propriedades específicas e fornece um contra exemplo para a Versão Modificada da Conjectura Discreta de Markus-Yamabe, para detalhes referentes à conjectura, ver [BS11].

Definição 3.4.1. Seja A uma matriz quadrada real. Definimos o espectro de A, denotado por $\operatorname{Spec}(A)$, como o conjunto de todos os autovalores de A. De forma análoga, se $h: \mathbb{R}^{2} \rightarrow \mathbb{R}^{2}$ é uma aplicação diferenciável, definimos $\operatorname{Spec}(h)$ como sendo o conjunto de todos os autovalores de Dh| $\left.\right|_{p}$, com $p$ variando em $\mathbb{R}^{2}$.

O principal resultado obtido em [BS11] é o seguinte Teorema:

Teorema 3.4.1. Existe um difeomorfismo $h \in \mathcal{H}$ de classe $C^{r}$ (para todo $r \geq 1$ ) satisfazendo a propriedade $\operatorname{spec}\left(\left.D h\right|_{p}\right) \subset B_{1}(0)$ para todo $x \in \mathbb{R}^{2}$ e que possui uma intersecção homoclínica transversal, e portanto, contém infinitos pontos periódicos.

Então, como intersecções homoclínicas são estáveis por $C^{1}$ pertubações, difeomorfismos genéricos satisfazendo as hipóteses do Teorema acima não são Morse-Smale, isto é, existe um conjunto aberto de difeomorfismos contendo ferraduras.

A seguir, esboçaremos a construção do exemplo de Bernado e Salvador.

Inicialmente, usando um resultado de Zenhder [Ze73], os autores mostraram que existe um difeomorfismo $h$ de classe $C^{r}$ que preserva área e que satisfaz:

- $h$ possui um ponto periódico hiperbólico com uma intersecção homoclínica transversal em $B_{1}(0)$.

- Para $r_{0} \geq 1$, cada círculo $C_{r_{0}}=\left\{(x, y) \in \mathbb{R}^{2}=x^{2}+y^{2}=r_{0}^{2}\right\}$ é fixado por $h$ e rotacionado por um ângulo que cresce uniformemente com a respeito a $r_{0}$ $\left(h\left(C_{r_{0}}\right)=C_{r_{0}}\right.$ e a dinâmica de $h$ em cada círculo é uma rotação rígida). 
- $\operatorname{Spec}(h) \subset S^{1}$.

Esta intersecção homoclínica é persistente no conjunto dos difeomorfismos planares com a topologia $C^{1}$. Então, para $\varepsilon>0$ suficientemente pequeno, o difeomorfismo

$$
g(x, y)=\frac{1}{1+\epsilon} h(x, y)
$$

pertence a $\mathcal{H}$, satisfaz $\operatorname{Spec}(g) \subset B_{1 / 1+\varepsilon}(0)$, e o ponto periódico com intersecção homoclínica em $\Sigma$ é mantido por $g$.

Este difeomorfismo $g$ possui infinitos pontos periódicos hiperbólicos em $B_{1}$.

\subsection{Demonstração do Teorema 3.1.4}

Dado $\alpha \in[0,1)$ com $\alpha$ irracional, iremos apresentar um exemplo de um homeomorfismo $h_{\alpha} \in \mathcal{H}$ com o número de rotação de fins primos $\rho\left(h_{\alpha}, U\right)=\alpha$ e que possui um ponto periódico de sela com intersecção homoclínica em $\Sigma$, logo possui infinitos pontos periódicos. Este exemplo irá responder positivamente à pergunta 3.1.2.

Para construir tal homeomorfismo, utilizaremos os exemplos de Matsumoto em [Mat13] juntamente com o exemplo de Bernado e Salvador em [BS11], apresentados nas seções 3.3 e 3.4, respectivamente.

Considere $g: \mathbb{R}^{2} \rightarrow \mathbb{R}^{2}$ o homeomorfismo construído por Bernado e Salvador. O cilindro $\mathbb{A}=S^{1} \times(0, \infty)$ é identificado com $\mathbb{R}^{2} \backslash\{0\}$, de maneira que o fim inferior do cilindro $S^{1} \times\{0\}$ é identificado com a origem. Então, $g$ induz um homeomorfismo que preserva orientação em $\mathbb{A}$, que também chamaremos de $g$.

Dado $\alpha$ irracional, tomemos $h: \mathbb{A} \rightarrow \mathbb{A}$ o homeomorfismo construído por Matsumoto de modo que o número de rotação $\rho(h, U)=\alpha$, com $U$ a bacia de repulsão do infinito por $h$. Para este $\alpha$, definiremos um homeomorfismo $h_{\alpha}: \mathbb{A} \rightarrow \mathbb{A}$ do seguinte modo:

- Em $S^{1} \times(0,2]$, defina $h_{\alpha}=g$. Então, pela definição de $g$, para $t \in[1,2]$, temos que 


$$
h_{\alpha}(\theta, t)=\left(R_{\beta_{t}}(\theta), \frac{t}{1+\varepsilon}\right)
$$

com $R_{\beta_{t}}$ uma rotação de ângulo $\beta_{t}$ que cresce uniformemente com a respeito a $t$.

- $\operatorname{Em} S^{1} \times[5, \infty)$, defina $h_{\alpha}=h$, onde

$$
h(\theta, t)=\left(f_{\alpha}(\theta), t-g_{0}(\theta, t)\right)
$$

$f_{\alpha}(\theta)$ é um difeomorfismo de Denjoy com número de rotação $\alpha$, tal que $C_{\alpha}$ tem medida 0 e $g_{0}(\theta, t) \in[0,1]$ é uma função contínua. Aqui, lembre que das hipóteses satisfeitas por $g_{0}$, vale $h_{\alpha}\left(S^{1} \times\{5\}\right)=S^{1} \times\{4\}$.

- Finalmente, em $S^{1} \times[2,5]$, definimos $h_{\alpha}$ como

$$
h_{\alpha}(\theta, t)=\left(\varphi_{t}(\theta), \gamma(t)\right)
$$

onde, para $t \in[2,5]$,

$$
\gamma^{\prime}(t) \in[\delta, 1), \quad \gamma(2)=\frac{2}{1+\varepsilon} \text { e } \gamma(5)=4
$$

para algum $\delta>0$. Além disso, $\varphi_{t}: S^{1} \rightarrow S^{1}$ é uma família de difeomorfismos $C^{1}$ tal que $h_{\alpha}$ é uma colagem $\left.C^{1} \operatorname{com} h_{\alpha}\right|_{S^{1} \times(0,2]}$ e uma colagem $\left.C^{0} \operatorname{com} h_{\alpha}\right|_{S^{1} \times[5, \infty)}$.

Definida desse modo, resulta que $h_{\alpha}$ é um homeomorfismo do plano com $\left.h_{\alpha}\right|_{S^{1} \times(0,5)}$ : $S^{1} \times(0,5) \rightarrow S^{1} \times(0,4)$ um difeomorfismo de classe $C^{1}$. Além disso, por construção, segue que acima de $S^{1} \times\{2\}, h_{\alpha}$ possui um único conjunto minimal $C_{\alpha} \subset S^{1} \times\{10\}$.

Agora, note que as condições impostas à função $\gamma$ implicam que acima de $S^{1} \times\{1\}$, $\Sigma$ é uma união de "cabelos" disjuntos que têm origem no conjunto $C_{\alpha}$ e que estão se acumulando no atrator do exemplo de Bernado e Salvador. Isto porque, definindo $\Lambda=\Sigma \cap\left(S^{1} \times[5,10]\right)$, resulta do exemplo de Matsumoto que $\Lambda$ é um conjuntos de segmentos de retas verticais com origem no conjunto $C_{\alpha}$, donde $\Lambda=C_{\alpha} \times[5,10]$. Além disso, como $C_{\alpha}$ é um conjunto invariante, pela definição de $h_{\alpha}$, existe $n \in \mathbb{N}$ tal que $\Sigma \cap\left(S^{1} \times[1, \infty)\right) \subset h_{\alpha}^{n}(\Lambda)$. Observe a figura 3.6 para ter uma ideia do que está acontecendo. 


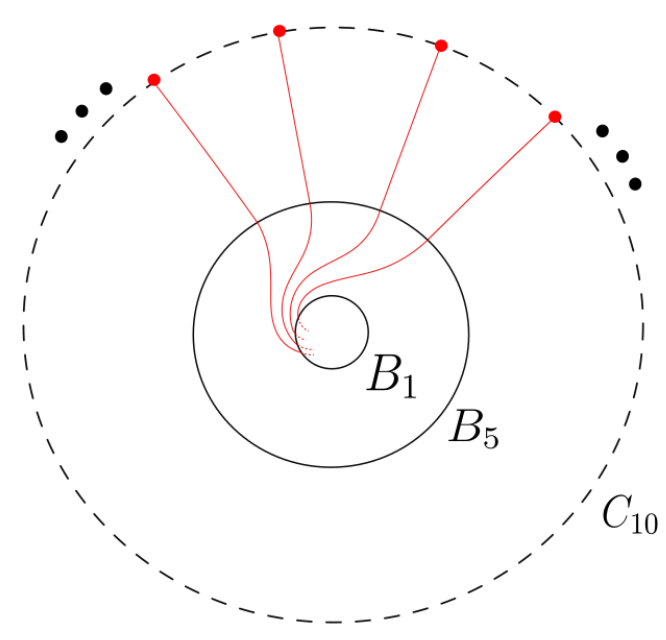

Figura 3.6: Em vermelho, temos os "cabelos" de $\Sigma$ com origem nos pontos do conjunto minimal $C_{\alpha}$ e que estão se enrolando em torno do atrator do exemplo de Bernado e Salvador.

Como $C_{\alpha}$ tem medida de Lebesgue igual a zero, $m\left(C_{\alpha}\right)=0$, no próximo Lema mostraremos que $m(\Sigma)=0$, donde conclui-se que $h_{\alpha} \in \mathcal{H}$. Para demonstramos este lema, será usado a seguinte proposição (Proposição 10.13 em [Is09]).

Proposição 3.5.1. Denote por $m$ a medida de Lebesgue. Dados $A, B \subset \mathbb{R}$ conjuntos mensuráveis e $\sigma$-finitos relativamente à $m$. Então $A \times B$ é mensurável, $\sigma$-finitos relativamente à $m$ e temos que $m(A \times B)=m(A) \times m(B)$.

Lema 3.5.1. Considere o homeomorfismo $h$ definido acima. Como $m\left(C_{\alpha}\right)=0$, então $m(\Sigma)=0$.

Demonstração. Seja $\Lambda=C_{\alpha} \times[5,10]$. Como $m\left(C_{\alpha}\right)=0$, pela Proposição 3.5.1, notemos que $m(\Lambda)=0$. Consequentemente, como $\Sigma \cap\left(S^{1} \times[1, \infty)\right) \subset h^{n}(\Lambda)$ para algum $n \in \mathbb{N}$, temos que acima de $S^{1} \times\{1\}, \Sigma$ tem medida de Lebesgue zero. Portanto,

$$
m(\Sigma)=m\left(\Sigma \cap\left(S^{1} \times(0,1)\right)\right)
$$

Agora, denotando por $A=\left\{x \in \Sigma: h_{\alpha}^{-n}(x) \in S^{1} \times(0,1) \forall n \in \mathbb{N}\right\}$, observemos que $A$ é o atrator para $g$ do exemplo de Bernado e Salvador. Então, como $\operatorname{Spec}(g) \subset B_{1 / 1+\varepsilon}(0)$, 
resulta que $m(A)=0$. Logo, supondo por absurdo que $m(\Sigma)>0$, obtemos que existe um subconjunto $B \subset \Sigma \cap\left(S^{1} \times(0,1)\right)$ com $m(B)>0$ e existe $n>0$ de modo que

$$
h_{\alpha}^{-n}(B) \cap\left(S^{1} \times[1, \infty)\right)
$$

tem medida positiva. Como isto é um absurdo, o Lema segue.

Nos falta apenas justificar o porquê $\rho\left(h_{\alpha}, U\right)=\alpha$. Com esta finalidade, vejamos que no Teorema 3.1.3, para mostrar que o número de rotação $\rho\left(h, U_{\infty}\right)=\alpha$, Matsumoto utilizou apenas que os pontos de $C_{\alpha}$ são acessíveis pela bacia $U=U_{\infty}$. Logo, os pontos de $C_{\alpha}$ estão associados a fins primos acessíveis e tais fins primos possuem número de rotação $\alpha$. Portanto, como os pontos acessíveis de $C_{\alpha}$ são preservados por $h_{\alpha}$, resulta que o número de rotação $\rho\left(h_{\alpha}, U\right)=\alpha$ é irracional.

Note que que os "cabelos" que têm origem no conjunto $C_{\alpha}$ estão se acumulando nos fechos das variedades instáveis das selas periódicas presentes em $S^{1} \times(0,1)$ de tal modo que estes pontos de sela não são acessíveis por $U$. Será mostrado no próximo capítulo que $\mathcal{W}^{u}(p) \subset \partial U$ para todo ponto periódico $p \in \partial U$ de sela e que a irracionalidade do número de rotação $\rho(h, U)$ implica que não pode existir ponto acessível em qualquer ramo instável de pontos de sela. 


\section{Capítulo 4}

\section{Alguns Resultados 2}

Este capítulo está dividido da seguinte forma: na primeira seção, apresentaremos algumas propriedades que serão usadas nas seções posteriores, na segunda seção definiremos o que é uma ferradura de Smale e mostraremos que tais ferraduras têm número de rotação de fins primos racional e, na terceira seção, exibiremos propriedades dos difeomorfismos $h \in \mathcal{H}$, quando o número de rotação de fins primos $\rho(h, U)$ é irracional. Estes são os principais resultados obtidos nesta tese.

\subsection{Preliminares}

Dado $h \in \mathcal{H}$, considere $U$ a bacia de repulsão do infinito e denote por $\Sigma=\partial U$.

Usando o exemplo de Addas Zanata e Veiga Gomes e as ideias do trabalho de Matsumoto, construímos na seção 3.5 um exemplo de um homeomorfismo $h \in \mathcal{H}$ com número de rotação de fins primos $\rho(h, U)$ irracional, de modo que existe um ponto periódico $p \in \Sigma$ de sela e tal que as variedades instável e estável de $p$ se intersectam transversalmente. No entanto, este homeomorfismo apresenta a propriedade importante de que não existem pontos acessíveis em $\overline{W^{u}(p)}$ (será provado na Proposição 4.1.1 que $\left.\overline{W^{u}(p)} \subset \Sigma\right)$. Em particular, temos que $\Sigma \backslash \overline{W^{u}(p)} \neq \emptyset$.

Em vista de tal exemplo que foi construído, agora, estaremos interessados em estudar 
a Pergunta 3.1.2 com a hipótese adicional de que $\Sigma=\overline{W^{u}(p)}$, ou seja, se $p \in \Sigma$ um ponto de sela com intersecção homoclínica transversal, queremos responder a pergunta:

Pergunta 4.1.1. Se $h \in \mathcal{H}$ é difeomorfismo de classe $C^{1}$, é possível que o número de rotação $\rho(h, U)$ seja irracional e exista ponto de sela $p$ com intersecção homoclínica transversal tal que $\Sigma=\overline{\mathcal{W}^{u}(p)}$ ?

No que segue, vamos considerar $h \in \mathcal{H}$, que existe um ponto fixo $p \in \Sigma$ de sela com intersecção homoclínica transversal e vamos supor que os autovalores de $D h(p)$ são ambos positivos. A próxima proposição fornece algumas informações referentes às variedades $\mathcal{W}^{s}(p)$ e $\mathcal{W}^{u}(p)$

Proposição 4.1.1. Dado $h \in \mathcal{H}$, seja $p \in \Sigma$ um ponto fixo de sela com intersecção homoclínica transversal. Então,

(i) A variedade instável $\mathcal{W}^{u}(p)$ está inteiramente contida em $\Sigma$ e $\overline{\mathcal{W}^{u}(p)} \subset \Sigma$, porque $\Sigma$ é compacto.

(ii) A variedade estável $\mathcal{W}^{s}(p)$ não pode estar totalmente contida em $\Sigma$.

(iii) Se existir ponto acessível na variedade $\mathcal{W}^{u}(p)$, então o número de rotação $\rho(h, U)$ é racional.

Demonstração. (i) Como $U$ é a bacia de repulsão do infinito, dado $x \in U$, note que

$$
\lim _{n \rightarrow \infty} h^{-n}(x) \rightarrow \infty
$$

Por outro lado, se $x \in \mathcal{W}^{u}(p)$, temos que $h^{-n}(x) \rightarrow p$ quando $n \rightarrow \infty$. Consequentemente, $\mathcal{W}^{u}(p) \subset \Sigma$. Sendo $\Sigma$ um conjunto fechado, $\overline{\mathcal{W}^{u}(p)} \subset \Sigma$.

(ii) Iremos mostrar que se $\mathcal{W}^{s}(p) \subset \Sigma$ então $\Sigma^{c}=U$ não pode ser conexo. De fato, como $\Sigma$ possui interior vazio e pelo item $(i)$ temos que $\mathcal{W}^{u}(p) \subset \Sigma$, tomando qualquer $x \in \mathcal{W}^{s}(p) \cap \mathcal{W}^{u}(p)$, observemos que, caso $\mathcal{W}^{s}(p) \subset \Sigma$, então

$$
\left(\left.\left.\mathcal{W}^{s}(p)\right|_{[x, h(x)]} \cup \mathcal{W}^{u}(p)\right|_{[x, h(x)]}\right)^{c}
$$


possui mais de uma componente conexa, onde

$$
\left.\mathcal{W}^{s}(p)\right|_{[x, h(x)]} \text { e }\left.\mathcal{W}^{u}(p)\right|_{[x, h(x)]}
$$

representam os "arcos" de variedade estável e instável de $x$ até $h(x)$, respectivamente. Portanto, se $\mathcal{W}^{s}(p) \subset \Sigma$, temos que $\Sigma^{c}$ não é conexo. No entanto, como $\Sigma^{c}=U$ é conexo pois é a bacia de repulsão do infinito, chegamos que $\mathcal{W}^{s}(p)$ não pode estar totalmente contida em $\Sigma$.

(iii) Suponhamos que exista um ponto acessível $a \in \mathcal{W}^{u}(p)$. Como $h(a)$ é acessível e o aberto $U$ é conexo, considere $\sigma_{a} \subset U$ um crosscut que com pontos finais em $a$ e $h(a)$. Pelo Lema 2.2.2, temos que $\left(\Sigma \cup \sigma_{a}\right)^{c}$ possui exatamente duas componentes conexas. Sejam $D_{1}$ e $D_{2}$ estas componentes conexas.

Observe que, para uma dessas, digamos $D_{1}$, temos $p \notin \overline{D_{1}}$. De fato, definindo $J=\left.\mathcal{W}^{u}(p)\right|_{[a, h(a)]} \cup \sigma_{a}$, como $J$ é uma curva fechada simples, pelo Teorema da curva de Jordan, obtemos que existem componentes conexas abertas $A_{1}$ e $A_{2}$, tais que $J^{c}=A_{1} \cup A_{2}$. Além disso,

$$
\partial A_{i}=J, \text { para } i=1,2 \text {. }
$$

Então, como $p \notin J$, temos que para algum $A_{i}$, digamos $A_{1}, p \notin \overline{A_{1}}$. Portanto, como

$$
\left(\Sigma \cup \sigma_{a}\right)^{c} \subset A_{1} \cup A_{2}
$$

considerando que $D_{1} \subset A_{1}$, obtemos que $p \notin \overline{D_{1}}$.

Agora, considere $\varepsilon>0$ satisfazendo

$$
\overline{D_{1}} \cap B_{\varepsilon}(p)=\emptyset
$$

Pelo item $(i i)$, a variedade $\mathcal{W}^{s}(p)$ não pode estar inteiramente contida em $\Sigma$. Logo, podemos tomar um $x \in \mathcal{W}^{s}(p) \cap U$ e percorrer $\mathcal{W}^{s}(p)$, por um dos lados de $x$, até o primeiro momento em que $\mathcal{W}^{s}(p) \cap \Sigma \neq \emptyset$. Isto mostra que o conjuntos dos pontos acessíveis em $\mathcal{W}^{s}(p) \cap \Sigma$ é não vazio e podemos tomar os caminhos acessando tais pontos de modo que estejam contidos em $\mathcal{W}^{s}(p)$. 
Então, considere $m \in \mathcal{W}^{s}(p) \cap \Sigma$ um ponto acessível com $\gamma:[0,1) \rightarrow \mathcal{W}^{s}(p) \cap U$ um endcut acessando o ponto $m$. Como $\gamma \subset \mathcal{W}^{s}(p)$, existe $n_{0}$ suficientemente grande tal que, para $n \geq n_{0}$,

$$
h^{n}(\gamma) \subset B_{\varepsilon}(p)
$$

Consequentemente, $h^{n}(\gamma) \cap \overline{D_{1}}=\emptyset$, para todo $n \geq n_{0}$. Isto contradiz o Lema 2.3.1.

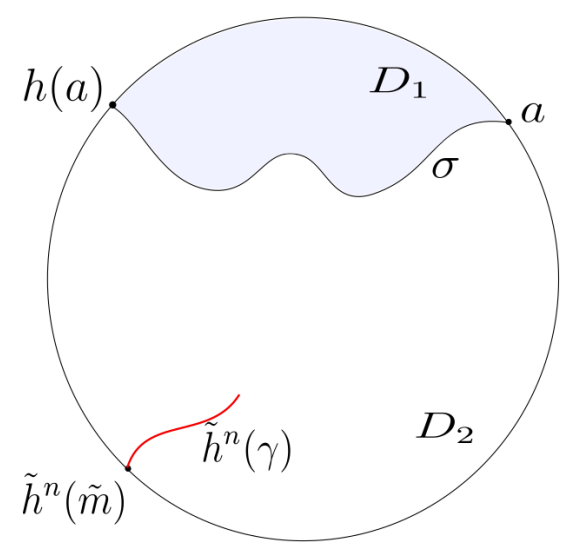

Figura 4.1: Informações na compactificação de fins primos de $U$.

Uma consequência imediata da Proposição anterior é o seguinte resultado.

Corolário 4.1.1. Dada $h \in \mathcal{H}$, se o número de rotação $\rho(h, U)$ for irracional, então não existe ponto acessível na variedade instável de nenhum ponto periódico de sela em $\Sigma$.

Observação 4.1.1. Uma primeira consequência que surge quando assumimos que o número de rotação $\rho(h, U)$ é irracional e $\Sigma=\overline{\mathcal{W}^{u}(p)}$ resulta do Corolário 4.1 .1 e afirma que não pode existir ponto acessível na variedade instável de qualquer ponto de sela em $\Sigma$. Além disso, se h é um difeomorfismo de classe $C^{1}$, usando o Teorema 2.1.3 devido a Pixton, podemos tomar h num conjunto genérico tal que, se o fecho de um ramo de $\mathcal{W}^{u}(p)$ intersecta um ramo de $\mathcal{W}^{s}(p)$, então estes ramos se intersectam transversalmente. Mais 
ainda, nestas hipóteses, esta mesma condição genérica garante que a variedade $\mathcal{W}^{u}(p)$ intersecta transversalmente os dois ramos estáveis de todo ponto periódico de sela em $\Sigma$, pois, caso contrário, existiria uma sela periódica acessivel e, portanto, número de rotação $\rho(h, U)$ seria racional.

Observação 4.1.2. Considere $h \in \mathcal{H}$ de Classe $C^{1}$. Sejam $\mathcal{W}_{1}^{u}(p)$ e $\mathcal{W}_{2}^{u}(p)$ os dois ramos instáveis de $\mathcal{W}^{u}(p)$. Supondo que $\mathcal{W}_{i}^{u}(p) \cap \mathcal{W}^{s}(p) \neq \emptyset$, então o $\lambda$-lema nos permite concluir que $\overline{\mathcal{W}_{i}^{u}(p)}=\Sigma$, pois $\overline{\mathcal{W}^{u}(p)}=\Sigma$. Agora, vamos supor que o ramo $\mathcal{W}_{1}^{u}(p)$ intersecta transversalmente um ramo de $\mathcal{W}^{s}(p)$ e supor que o ramo $\mathcal{W}_{2}^{u}(p)$ não intersecta $\mathcal{W}^{s}(p)$. Como estamos supondo que o número de rotação $\rho(h, U) \in \mathbb{Q}^{c}$, pela observação 4.1.1, segue que, $\mathcal{W}_{1}^{u}(p)$ precisa intersectar transversalmente os dois ramos de $\mathcal{W}^{s}(p)$.

\subsection{Número de Rotação da Ferradura de Smale}

Existem muitos exemplos de difeomorfismos $h \in \mathcal{H}$ tais que $h$ possui um ponto fixo $p \in \Sigma$ de sela com intersecção homoclínica $C^{1}$-transversa. Talvez o mais clássico deles seja a própria ferradura construída por Smale em 1965, que definiremos a seguir. A ferradura de Smale é uma transformação topológica que fornece uma base para o entendimento das propriedades caóticas dos sistemas dinâmicos. Mostraremos nesta seção que o número de rotação $\rho(h, U)$ é racional quando $h$ é uma ferradura de Smale.

Definição 4.2.1. Considere um difeomorfismo $h \in \mathcal{H}$. Diremos que $h$ é uma ferradura de Smale, quando existirem um quadrado fechado $Q$ centrado na origem e de lado l, um semidisco inferior $A$ de raio l/2 localizado abaixo da base de $Q$ e $B$ um semi-disco superior de raio $l / 2$ localizado acima de topo de $Q$, de modo que, chamando de $N=Q \cup A \cup B$ o disco topológico obtido pela união dessas três regiões, temos que:

(i) Para todo $x \in \mathbb{R}^{2} \backslash N$, existe $n \in \mathbb{N}$ satisfazendo $h^{n}(x) \in N$.

(ii) $h(N) \subset \operatorname{int} N$, com $h(A \cup B) \subset \operatorname{int}(A \cup B)$.

(iii) A dinâmica de $h$ em $Q \cap h^{-1}(Q)$ satisfaz: $h$ comprime horizontalmente $Q$ por um fator $0<\lambda<1$ e expande verticalmente por um fator $\mu>1$. 
(iv) A intersecção $h(Q) \cap Q$ possui um número $m$ retângulos disjuntos, todos com mesma largura e com $m$ satisfazendo $2 \leq m<\infty$.

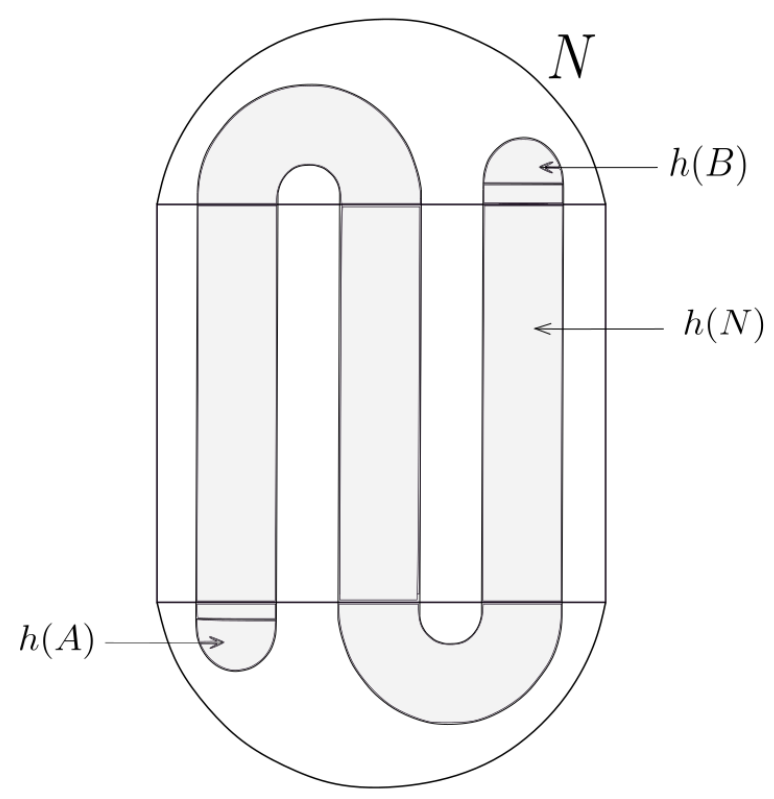

Figura 4.2: Exemplo em que a intersecção $h(Q) \cap Q$ possui 3 retângulos

Observação 4.2.1. Em particular, vale uma condição mais forte em (ii), $h$ possui um ou dois pontos periódicos atratores em $A \cup B$. Logo, os demais pontos periódicos de $h$ estão contidos no retângulo $Q$. Como na definição de $h, h(U) \subset U$, segue que $h^{2}(N) \subset h(N) \subset N$. Além disso, se $h(Q) \cap Q$ possui $m$ retângulos de largura $\lambda$, então $h^{2}(Q) \cap Q$ consiste de $m^{2}$ retângulos disjuntos e de largura $\lambda^{2} l$.

Para o entendimento de algumas propriedades das ferraduras de Smale, um importante espaço métrico e o espaço das sequências de $m$ símbolos $\Sigma_{m}$ que será definido a seguir.

Definição 4.2.2. O espaço das sequências de $m$ símbolos, $\Sigma_{m}$, é definido por

$\Sigma_{m}=\{0,1, \ldots, m-1\}^{\mathbb{Z}}=\left\{s=\left(\ldots, s_{-2}, s_{-1}, s_{0}, s_{1}, s_{2}, \ldots\right) \mid s_{j} \in\{0,1, \ldots m-1\}, j \in \mathbb{Z}\right\}$. 
Uma métrica em $\Sigma_{m}$ é definida por

$$
d(s, t)=\sum_{-\infty}^{\infty} \frac{\left|s_{j}-t_{j}\right|}{2^{|j|}},
$$

onde $s=\left(s_{j}\right)_{j \in \mathbb{Z}}, t=\left(t_{j}\right)_{j \in \mathbb{Z}}$ e $s_{j}, t_{j} \in\{0,1, \ldots, m-1\}$ para todo $j \in \mathbb{Z}$.

Um fato que segue direto da Definição 4.2.2 é que essa métrica faz de $\Sigma_{m}$ um espaço métrico.

Definição 4.2.3. A aplicação shift (ou shift bilateral) sobre $\Sigma_{m}$ é definida por $\sigma(s)=t$, onde $t_{k}=s_{k+1}$, para $t=\left(t_{k}\right)_{k \in \mathbb{Z}}$ e $s=\left(s_{k}\right)_{k \in \mathbb{Z}}$ em $\Sigma_{m}$. O espaço $\Sigma_{m}$ junto com a aplicação $\sigma$ é chamado shift bilateral.

Suponha que um difeomorfismo $h \in \mathcal{H}$ satisfaz as propriedades da Definição 4.2.1 e que $m$ representa o número de retângulos da intersecção $h(Q) \cap Q$. Representando por $\Lambda$ o conjunto de todos os pontos cujas órbitas positivas e negativas permanecem no quadrado $Q$, é um fato conhecido que a aplicação $h: \Lambda \rightarrow \Lambda$ é topologicamente conjugada a aplicação shift $\sigma: \Sigma_{m} \rightarrow \Sigma_{m}$. Então, como a aplicação shift $\sigma: \Sigma_{m} \rightarrow \Sigma_{m}$ possui exatamente $m$ pontos fixos, que são os pontos

$$
s=\left(\ldots, s_{0}, s_{0}, s_{0}, s_{0}, s_{0}, \ldots\right), \operatorname{com} s_{0} \in\{0,1, \ldots, m-1\},
$$

temos que $\left.h\right|_{\Lambda}$ possui $m$ pontos fixos, ou seja, existem apenas $m$ pontos fixos de sela em $\Sigma \cap Q$.

Dado um ponto fixo de sela $p \in \Sigma$, temos que a variedade estável local do ponto $p$ é o segmento de reta horizontal contido no retângulo $Q$ e que passa por $p$. De forma análoga a variedade instável local de ponto $p$ é o segmento de reta vertical contido em $Q$ e que passa por esse ponto.

Construído dessa forma, uma ferradura de Smale $h$ possui pontos fixos de sela com interseção homoclínica. Para um tal ponto fixo de sela $p \in \Sigma$, vale que $\Sigma=\overline{\mathcal{W}^{u}(p)}$. Além disso, para todo $n \in \mathbb{N}$, temos que

$$
\overline{\mathcal{W}^{u}(p)} \cap Q \subset h^{n}(Q) \cap Q
$$


Então, dado $h \in \mathcal{H}$, supondo que $h$ é uma ferradura de Smale, para todo ponto fixo de sela $p \in \Sigma$, temos que $\Sigma=\overline{\mathcal{W}^{u}(p)}$. O seguinte resultado mostrará que ferraduras de Smale têm número de rotação $\rho(h, U)$ racional.

Teorema 4.2.1. Dado $h \in \mathcal{H}$, se $h$ é uma ferradura de Smale, então o número de rotação $\rho(h, U) \in \mathbb{Q}$.

Demonstração. Para provar este resultado, mostraremos que nas hipóteses deste Teorema, existe um ponto periódico acessível $q \in \Sigma$. Consequentemente, usando o Teorema 2.4.2, a existência deste ponto $q$ implicará que o número de rotação $\rho(h, U)$ é racional. Considere $p \in \Sigma$ um ponto fixo de sela. Então, $\Sigma=\overline{\mathcal{W}^{u}(p)}$. Inicialmente, notemos que quando um dos ramos de variedade $\mathcal{W}^{s}(p)$ não é intersectado por $\mathcal{W}^{u}(p)$, resulta da Observação 4.1 que o próprio ponto $p$ é um ponto periódico acessível, implicando que o número de rotação $\rho(h, U)$ é racional.

Vamos então considerar os casos em que os dois ramos da variedade $\mathcal{W}^{s}(p)$ são intersectados por ramos de $\mathcal{W}^{u}(p)$. Como $h$ é uma ferradura de Smale, então existe um quadrado $Q$ de lado $l$ satisfazendo a Definição 4.2.1. Assim, temos a união finita de retângulos,

$$
h(Q) \cap Q=\bigcup_{i=1}^{m} V_{i},
$$

todos com a mesma largura $\lambda_{0}$, onde $\lambda_{0}=\lambda l$. Note que, podemos assumir que $0<l<1$.

Para facilitar a notação, vamos assumir que o ponto $p$ é a origem. Então, as componentes conexas de $\mathcal{W}^{s}(0) \cap Q$ e $\mathcal{W}^{u}(0) \cap Q$ que contém a origem são, respectivamente, as componentes conexas do eixos cartesianos $x$ e $y$ restritos a $Q$.

Visto que a origem não é acessível, existe algum retângulo não extremo tal que a origem pertence a ele. Isto porque a variedade $\mathcal{W}^{u}(0)$ tem intersecção não vazia com os dois segmentos $\mathcal{W}^{s}(0) \cap\left\{(x, 0) \in \mathbb{R}^{2}: x<0\right\}$ e $\mathcal{W}^{s}(0) \cap\left\{(x, 0) \in \mathbb{R}^{2}: x>0\right\}$.

Os retângulos são todos verticais e disjuntos. Assim, ordenaremos os mesmos da esquerda para à direita, sendo $V_{1}$ o mais a esquerda e $V_{m}$, para algum $m \in \mathbb{N}$, o mais à 
direita. Isto define uma relação de ordem neste conjunto de retângulos. Utilizaremos a notação $\prec$ para representar esta relação de ondem e escrevemos $V_{1} \prec V_{2} \prec \cdots \prec V_{m}$.

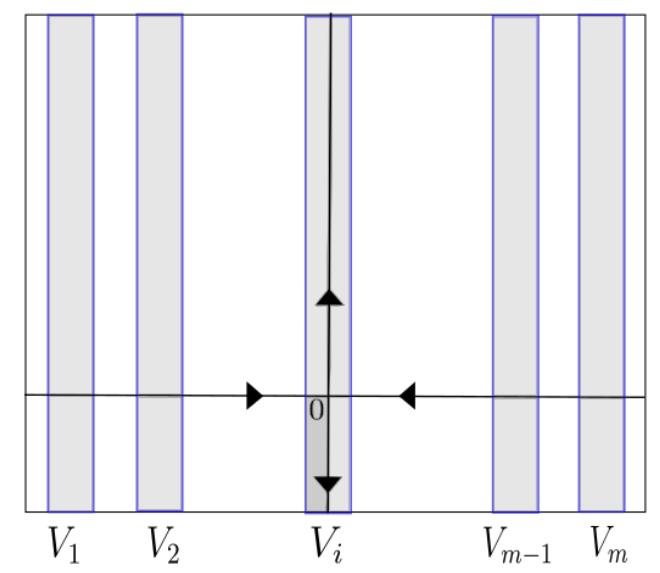

Figura 4.3: Informaçõs de $Q \cap h(Q)$ no plano.

Note que, para cada retângulo $V_{i}$, a intersecção $h^{2}(Q) \cap V_{i}$ possui $m$ retângulos disjuntos $V_{i j}$, com $1 \leq j \leq m$ e todos com largura $\lambda^{2} l$. Além disso, como $h$ preserva orientação, após definirmos a ordem $V_{1} \prec V_{m}$ dos retângulos de $h(Q) \cap Q$, existem apenas duas possibilidades para a ordem dos retângulos de $h^{2}(Q) \cap V_{i}$, a saber,

- $h^{2}(Q) \cap V_{i}$ tem a mesma ordem de $h(Q) \cap Q$, isto é, $V_{i 1} \prec V_{i m}$.

- $h^{2}(Q) \cap V_{i}$ reverte a ordem de $h(Q) \cap Q$, isto é, $V_{i m} \prec V_{i 1}$.

Logo, referente às ordens das intersecções $h^{2}(Q) \cap V_{1}$ e $h^{2}(Q) \cap V_{m}$, apenas um dos seguintes casos pode ocorrer:

(i) Ao menos uma destas intersecções tem a mesma ordem de $h(Q) \cap Q$, ou seja, sobra três casos:

$$
\begin{aligned}
& -V_{11} \prec V_{1 m} \text { e } V_{m 1} \prec V_{m m} \text {, ou } \\
& -V_{1 m} \prec V_{11} \text { e } V_{m 1} \prec V_{m m} \text {, ou }
\end{aligned}
$$


$-V_{11} \prec V_{1 m}$ e $V_{m m} \prec V_{m 1}$

(ii) Ambas as intersecções revertem a ordem de $h(Q) \cap Q$, ou seja,

$$
-V_{1 m} \prec V_{11} \text { e } V_{m m} \prec V_{m 1} \text {. }
$$

Provaremos que em ambos os casos $(i)$ e $(i i)$, existe ponto periódico acessível em pelo menos um dos retângulos $V_{1}$ e $V_{m}$.

Caso (i). Sem perda de generalidade, podemos assumir que $V_{m 1} \prec V_{m m}$. Por indução, obtemos que, para todo $n \in \mathbb{N}$,

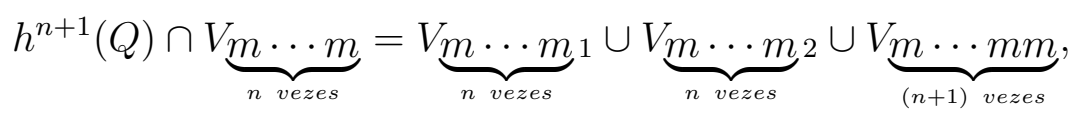

com a ordem

$$
V_{n \text { vezes }}^{m \cdots m} 1 \prec V_{(n+1) \text { vezes }}^{m \cdots m m} .
$$

Isto vai permitir concluir que, para todo $n \in \mathbb{N}$, a intersecção

$$
h^{n+1}(Q) \cap V_{n \text { vezes }}^{m \cdots m}
$$

tem a mesma ordem de $h(Q) \cap Q$. Logo, dado $n \in \mathbb{N}$, o retângulo extremo direito da intersecção $h^{n}(Q) \cap Q$ é o retângulo $C_{m}^{n}$, definido por

$$
C_{m}^{n}=V_{\underbrace{m \cdots m}_{\text {vezes }}}^{m .}
$$

Observe que o retângulo $C_{m}^{n}$ é formado pelos pontos $x \in Q$ tais que $h^{-j}(x) \in C_{m}^{n-j}$, para $1 \leq j \leq n$. Seja

$$
\Gamma=\bigcap_{n \geq 0} C_{m}^{n}
$$


Fato: $\Gamma$ é vertical em $Q$ e todo ponto de $\Sigma$ é acessível por semi-retas horizontais vindas da direita de $Q$. De fato, como cada retângulo $C_{m}^{n}$ é vertical e $C_{m}^{n} \rightarrow \Gamma$, segue que $\Gamma$ é vertical em $Q$. Agora, supondo que existe algum ponto de $\Gamma$ que não é acessível, note que devem existir pontos de $\Sigma$ à direta de $\Gamma$, mas como isto contradiz o fato que $\Sigma \cap Q \subset h^{n}(Q) \cap Q$ para todo $n$, obtemos que todos os pontos de $\Gamma$ são acessíveis.

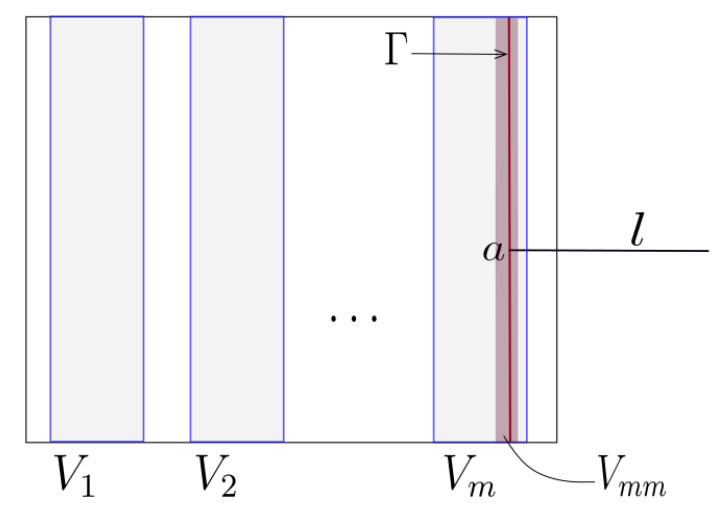

Figura 4.4: Semi-reta $l$ acessando o ponto $a \in \Gamma$.

Então, como existe um ponto fixo de sela $q \in C_{m}^{n}$, necessariamente, temos que $q \in \Gamma$. Isto conclui a demonstração de que existe sela acessível em $\Sigma$.

Caso (ii). Como o disto topológico $N$, na definição 4.2.1, satisfaz

$$
h^{2}(N) \subset \operatorname{int} h(N) \subset \operatorname{int} N
$$

definindo $g=h^{2}$, obtemos que

$$
\left[\bigcap_{n \in \mathbb{N}} g^{n}(N)\right] \cap Q=\left[\bigcap_{n \in \mathbb{N}} h^{n}(N)\right] \cap Q=\Sigma \cap Q
$$

Além disso, temos que $g(Q) \cap Q$ possui $m^{2}$ retângulos sendo que as intersecções $g^{2}(Q) \cap V_{1}$ e $g^{2}(Q) \cap V_{m^{2}}$ possuem a mesma ordem $g(Q) \cap Q$. Então, pelo caso $(i)$, sabemos que $g$ possui ponto fixo acessível em $Q$. 
Dado $h \in \mathcal{H}$ uma ferradura de Smale, a demonstração do Teorema 4.2.1 provou que existe ponto periódico acessível de sela $q \in \Sigma$. Mais ainda, mostrou que todos os pontos da variedade $\mathcal{W}^{u}(q)$ são acessíveis. O próximo Corolário, que segue imediatamente dos Teoremas 2.4.3 e 4.2.1, mostrará que que este fato não foi apenas uma coincidência e nos dirá onde estão todos os pontos acessíveis de $h$, isto porque os pontos periódicos de $h$ são pontos hiperbólicos.

Corolário 4.2.1. Nas hipóteses do Teorema 4.2.1, se h é uma ferradura de Smale, então todo ponto acessível ou é um ponto periódico ou está na variedade instável de um ponto periódico acessível.

O seguinte Corolário é consequência da demonstração do Teorema 4.2.1.

Corolário 4.2.2. Se $h \in \mathcal{H}$ é uma ferradura de Smale, então $h$ possui um fixo acessível ou um ponto acessível de período dois.

\subsection{Consequências do Número de Rotação de Fins Primos $\rho(h, U)$ Irracional.}

Nesta secção, consideremos que $h \in \mathcal{H}$ é um difeomorfismo de classe $C^{1}, U$ é a bacia de repulsão do infinito e $\Sigma=\overline{W^{u}(p)}$, com $p$ um ponto fixo de sela com intersecção homoclínica transversal tal que os autovalores de $D h(p)$ são ambos positivos. Mais ainda, assumiremos a seguinte condição que foi provada genérica na topologia $C^{r}$, para todo $r \geq 1$, em [Pi82] por Pixton:

Se $p, q \in \Sigma$ são pontos periódicos de sela e, para um ramo instável $\mathcal{W}_{i}^{u}(p)$ de $\mathcal{W}^{u}(p)$ e um ramo $\mathcal{W}_{j}^{s}(q)$ de $\mathcal{W}^{s}(q)$ vale que $\overline{\mathcal{W}_{i}^{u}(p)} \cap \mathcal{W}_{j}^{s}(q) \neq \emptyset$, então $\mathcal{W}_{i}^{u}(p)$ intersecta o ramo $\mathcal{W}_{j}^{s}(q)$ de forma $C^{1}$-transversa.

O Teorema 4.2.1 e o Corolário 4.2.1 forneceram informações sobre o número de rotação $\rho(h, U)$ para uma classe muito restrita de difeomorfismos que satisfazem $h$ uma ferradura 
de Smale. Com o objetivo de obtermos informações sobre o número de rotação $\rho(h, U)$ para qualquer difeomorfismo $h \in \mathcal{H}$ nas condições acima, vamos supor adicionalmente que $\rho(h, U)$ é irracional e estudar as consequências de assumir tal fato.

Em [Bar91], Barge usou a teoria de limite inverso para construir exemplos de homeomorfismos que estão na classe $\mathcal{H}$, possuem número de rotação de fins primos irracional e $\Sigma=\overline{\mathcal{W}^{u}(p)}$, para $p$ um ponto fixo de sela. Mesmo sendo apenas um homeomorfismo, os pontos de sela estão bem definidos. No entanto, tais exemplos, provavelmente, não tem a propriedade genérica de Pixton.

Para a próxima proposição, considere $a \in \Sigma$ um ponto acessível. Como $h(a)$ também é um ponto acessível e $U$ é um aberto conexo do plano, considere $\sigma_{a} \subset U$ um crosscut com extremos nos pontos $a$ e $h(a)$. Pelo Lema 2.2.2, sabemos que $\left(\Sigma \cup \sigma_{a}\right)^{c}$ possui exatamente duas componentes conexas abertas. Sejam $D_{1}$ e $D_{2}$ estas componentes. Então, $\partial D_{i} \subset \Sigma \cup \sigma_{a}$ para $i=1,2$. Defina

$$
K_{i}^{a}=\partial D_{i} \backslash \sigma_{a}, \quad \operatorname{com} i=1,2 .
$$

Veja que a definição de $K_{i}^{a}$ pode depender da escolha do crosscut $\sigma_{a}$. No entanto, para os resultados que apresentaremos, podemos tomar qualquer crosscut com extremos nos pontos $a$ e $h(a)$. Mas, se $a$ for o conjunto principal de apenas um fim primo, então esta definição não depende de $\sigma_{a}$, no sentido de que, para todo crosscut $\sigma_{a}$ com extremos em $a$ e $h(a)$, os continuos $K_{1}^{a}$ e $K_{2}^{a}$ serão as fronteiras das componente conexas de $\left(\Sigma \cup \sigma_{a}\right)^{c}$ restritas a $\Sigma$. Isto porque, dado um crosscut $\sigma_{a}$, temos que $\left.\partial D_{i}\right|_{\mathbb{P}(U)}$ em $U^{*}$ consiste de um dos dois intervalos determinados pelos fins primos $\tilde{a}$ e $\tilde{h}(\tilde{a})$, que estão associados aos pontos acessíveis $a$ e $h(a)$, respectivamente.

O Lema que segue mostrará, em particular, que para todo ponto acessível $a \in \Sigma$, o ponto fixo de sela $p \in K_{1}^{a} \cap K_{2}^{a}$ quando o número de rotação $\rho(h, U)$ é irracional.

Lema 4.3.1. Seja $a \in \Sigma$ é um ponto acessível. Se o número de rotação $\rho(h, U) \in \mathbb{Q}^{c}$, então existe $n \in \mathbb{N}$ tal que, para $i=1$ ou 2 ,

$$
\Sigma=\bigcup_{j=0}^{n} h^{-j}\left(K_{i}^{a}\right) .
$$


Demonstração. Considere $a \in \Sigma$ um ponto acessível. Provaremos que é possível obter um $n_{1}>0$ que satisfaz este lema para $K_{1}^{a}$. Visto que podemos repetir o mesmo processo e obter um $n_{2}$ que satisfaz o lema para $K_{2}^{a}$, para que exista um $n$ comum para $K_{1}^{a}$ e $K_{2}^{a}$, basta tomar o $n$ como o maior entre $n_{1}$ e $n_{2}$.

Como definido anteriormente, seja $\sigma_{a}$ um crosscut com pontos finais em $a$ e $h(a)$ e considere um endcut $\gamma_{a}:[0,1) \rightarrow U$ acessando $a$ com $\gamma([0,1)) \subset \sigma_{a}$. No conjunto de fins primos $U^{*}$, denote por $\tilde{a}$ o fim primo tal que $\gamma(t) \rightarrow \tilde{a}$ em $U^{*}$ quando $t \rightarrow 1^{-}$e denote por $\tilde{h}$ a extensão de $\left.h\right|_{U}$ para $U^{*}$. A fronteira de $D_{1}$ em $U^{*}$ consiste de $\sigma$ unido com um intervalo fechado $I \subset \mathbb{P}(U)$ determinado pelos fins primos $\tilde{a}$ e $\tilde{h}(\tilde{a})$.

Dado que o número de rotação $\rho(h, U)$ é irracional e o intervalo $I$ é um domínio fundamental de $\left.\tilde{h}\right|_{\mathbb{P}(U)}$, existe um número $n_{1}>0$ tal que

$$
\mathbb{P}(U) \subset \bigcup_{j=0}^{n_{1}} \tilde{h}^{-j}(I) .
$$

Agora, tome $z \neq a \in \Sigma$ um ponto acessível com $\alpha(t):[0,1) \rightarrow U$ um endcut que termina em $z$ e seja $\tilde{z}$ o fim primo associado a $z \operatorname{com} \alpha(t) \rightarrow \tilde{z}$ quando $t \rightarrow 1^{-}$em $U^{*}$. Como já foi mencionado, existe $k \operatorname{com} 0 \leq k \leq n_{1}$ tal que $\tilde{h}^{k}(\tilde{z}) \in I$ e, pela irracionalidade de $\rho(h, U)$, temos que $\tilde{h}^{k}(\tilde{z}) \neq\{\tilde{a}, \tilde{h}(\tilde{a})\}$. Por este motivo, como $h^{k}(\alpha(t))=\tilde{h}^{k}(\alpha(t)) \in D_{1}$ se $t<1$, obtemos que existe $\varepsilon>0$ tal que $h^{k}(\alpha([\varepsilon, 1))) \subset D_{1}$. Portanto $h^{k}(z) \in K_{1}^{a}$.

Em particular, isto implica que, dado qualquer ponto acessível $z \in \Sigma$, existe um inteiro $k$ com $0 \leq k \leq n_{1}$ de modo que $h^{k}(z) \in K_{1}^{a}$. Por esta razão, o conjunto

$$
\bigcup_{j=0}^{n_{1}} h^{-j}\left(K_{1}^{a}\right)
$$

contém todos os pontos acessíveis de $\Sigma$. Finalmente, como $K_{1}^{a}$ é um compacto e o conjunto dos pontos acessíveis é um conjunto denso em $\Sigma$, podemos concluir que

$$
\Sigma=\bigcup_{j=0}^{n_{1}} h^{-j}\left(K_{1}^{a}\right) .
$$


Observação 4.3.1. Na demonstração do Lema 4.3.1, foi usado apenas que o número de rotação $\rho(h, U)$ é irracional. O mesmo resultado continua válido para os casos que estão nas hipóteses do Lema 4.3.1 mas $\Sigma \backslash \overline{\mathcal{W}^{u}(p)} \neq \emptyset$, por exemplo, no exemplo construído na demonstração do Teorema 3.1.4.

Corolário 4.3.1. Seja $a \in \Sigma$ é um ponto acessível. Se o número de rotação $\rho(h, U)$ for irracional, então $p \in K_{1}^{a} \cap K_{2}^{a}$. Também temos que $K_{i}^{a}$ intersecta os dois ramos de $\mathcal{W}_{1}^{u}(p)$, para $i=1,2$. Consequentemente, se existir algum ponto acessível $a \in \Sigma$ tal que $p \notin K_{1}^{a} \cap K_{2}^{a}$, então $\rho(h, U) \in \mathbb{Q}$.

Demonstração. Como $p$ é um ponto fixo e existe $n$ como no Lema anterior, então

$$
p \in K_{i}^{a} \cup h^{-1}\left(K_{i}^{a}\right) \ldots \cup h^{-n}\left(K_{i}^{a}\right)
$$

para $i=1,2$. Em particular, $p \in K_{1}^{a} \cap K_{2}^{a}$. As outras afirmações também são imediatas, basta notar que cada ramo instável de $\mathcal{W}^{u}(p)$ também está contido em $K_{i}^{a} \cup h^{-1}\left(K_{i}^{a}\right) \ldots \cup$ $h^{-n}\left(K_{i}^{a}\right)$.

Com o intuito de estudar as consequências que resultam da proposição acima, vamos entender a topologia de $\Sigma$ em uma vizinhança do ponto $p$. Este estudo é iniciado com o lema a seguir.

Lema 4.3.2. Para $\varepsilon>0$ suficientemente pequeno, a componente conexa de $\Sigma \cap B_{\varepsilon}(p)$ que contém $p$ é exatamente a componente conexa de $\mathcal{W}^{u}(p) \cap B_{\varepsilon}(p)$ que contém $p$.

Demonstração. Usando o Teorema 2.1.1 de Hatman-Grobman, podemos assumir que $h$ se escreve como $h(x, y)=(\mu x, \lambda y)$ em uma vizinhança aberta $V$ de $p$. Então, fixando um $\varepsilon>0$ suficientemente pequeno tal que a bola $B_{\varepsilon}(p)$ esteja contida em $V$, podemos assumir que, neste sistema de coordenas, $p$ é a origem e a componente conexa de $\mathcal{W}^{u}(p) \cap B_{\varepsilon}(p)$ que contém o ponto $p$ é o intervalo $I_{\varepsilon}$, obtido da intersecção do eixo das ordenadas com a bola $B_{\varepsilon}(p)$.

Agora, se $J_{\varepsilon}$ é a componente conexa de $\Sigma \cap B_{\varepsilon}(p)$ que contém $p$, veja que, por definição, $J_{\varepsilon} \supset I_{\varepsilon}$. No entanto, supondo por absurdo que $J_{\varepsilon} \neq I_{\varepsilon}$, então deve existir $r \in J_{\varepsilon}$ tal 
que $r \notin I_{\varepsilon}$. Como estamos supondo que $\rho(h, U)$ é irracional, a Observação 4.1.1 afirma que os dois ramos estáveis de $\mathcal{W}^{s}(p)$ são intersectados por $\mathcal{W}^{u}(p)$. Logo, com o $\lambda$-Lema, obtemos $I_{\varepsilon}$ é acumulado pela variedade $\mathcal{W}^{u}(p)$ pelos dois lados. Consequentemente, existe $r_{0} \in J_{\varepsilon} \cap \mathcal{W}^{u}(p)$ com $r_{0} \notin \xi_{\varepsilon}$. Mas note que isto implica que $\left(J_{\varepsilon} \cup \Sigma\right)^{c}$ não é conexo. Como isto é um absurdo, o lema segue.

Dado $a \in \Sigma$ um ponto acessível, considere os continuos $K_{1}^{a}$ e $K_{2}^{a}$ como definidos anteriormente. Usando os resultados acima, iremos mostrar agora que, sob certa hipótese genérica, se o número de rotação $\rho(h, U)$ é irracional, então $K_{1}^{a}=K_{2}^{a}=\Sigma$. Isso mostrará que no Lema 4.3.1, $n=0$.

Teorema 4.3.1. Considere $h \in \mathcal{H}$ e $U$ a bacia de repulsão do infinito. Suponhamos que $h$ satisfaz a condição genérica de Pixton, que o número de rotação $\rho(h, U)$ é irracional e $\Sigma=\overline{\mathcal{W}^{u}(p)}$, para $p$ um ponto fixo de sela. Então, para todo ponto acessível $a \in \Sigma$, temos que $K_{1}^{a}=K_{2}^{a}=\Sigma$.

Demonstração. Seja $a \in \Sigma$ um ponto acessível. Visto que o número de rotação $\rho(h, U)$ é irracional, o Corolário 4.3.1 mostra que

$$
p \in K_{1}^{a} \cap K_{2}^{a}, K_{i}^{a} \cap \mathcal{W}_{1}^{u}(p) \neq \emptyset \text { e } K_{i}^{a} \cap \mathcal{W}_{2}^{u}(p) \neq \emptyset .
$$

Para simplificar a notação, vamos considerar $K$ um continuo que satisfaz as mesmas hipóteses de $K_{1}^{a}$ e $K_{2}^{a}$, ou seja, $p \in K, a \in K, K \cap \mathcal{W}_{1}^{u}(p) \neq \emptyset$ e $K \cap \mathcal{W}_{2}^{u}(p) \neq \emptyset$. Inicialmente, provemos a seguinte afirmação:

Afirmação 1: Se $K$ intersecta algum ramo da variedade $\mathcal{W}^{u}(p)$, então $K$ contém o fecho de tal ramo.

Antes de iniciarmos a demonstração desta afirmação observemos que a demonstração deste Teorema é uma consequência imediata de tal afirmação. De fato, como o número de rotação $\rho(h, U)$ é irracional, segue da observação 4.1.2 que o fecho de pelo menos um dos ramos de $\mathcal{W}^{u}(p)$ é igual a $\Sigma$. Logo, assumindo a afirmação anterior, como $K \cap \mathcal{W}_{1}^{u}(p) \neq \emptyset$ e $K \cap \mathcal{W}_{2}^{u}(p) \neq \emptyset$, concluiremos que 


$$
\overline{\mathcal{W}_{1}^{u}(p)} \subset K \text { e } \overline{\mathcal{W}_{2}^{u}(p)} \subset K
$$

donde seguirá que $K=\Sigma$. Por este motivo, para concluirmos a demonstração deste Teorema, precisamos apenas provar a afirmação acima.

Prova da Afirmação. Sem perda de generalidade, vamos supor por absurdo que o ramo $\mathcal{W}_{1}^{u}(p)$ de $\mathcal{W}^{u}(p)$ é tal que

$$
\mathcal{W}_{1}^{u}(p) \cap K \neq \emptyset, \quad \operatorname{mas} \mathcal{W}_{1}^{u}(p) \nsubseteq K
$$

Como feito na demonstração do Lema 4.3.2, dado $\varepsilon>0$ suficientemente pequeno, denote por $I_{\varepsilon}$ a componente conexa de $\Sigma \cap B_{\varepsilon}(p)$ que contém $p$. Visto que $p \in K, a \in K$ e $K \subset \Sigma$, se necessário, podemos considerar um $\varepsilon$ menor e usar o mesmo lema para concluir que se $I_{\varepsilon_{k}}$ é a componente conexa de $K \cap B_{\varepsilon}(p)$ que contém $p$, então

$$
I_{\varepsilon_{k}}=I_{\varepsilon} \text { ou } I_{\varepsilon_{k}}=I_{\varepsilon} \cap \mathcal{W}_{1}^{u}(p)
$$

Se $I_{\varepsilon_{k}}=I_{\varepsilon}$, então $K \cap B_{\varepsilon_{k}}(p)$ intersecta os dois ramos de $\mathcal{W}^{u}(p)$, caso contrário, intersecta apenas um ramo. Considere a parametrização de $\mathcal{W}_{1}^{u}(p)$,

$$
\begin{aligned}
l:[0, \infty) & \longrightarrow \mathcal{W}_{1}^{u}(p) \\
s & \longmapsto l(s) .
\end{aligned}
$$

Observe que, como $I_{\varepsilon_{k}}=I_{\varepsilon}$ ou $I_{\varepsilon_{k}}=I_{\varepsilon} \cap \mathcal{W}_{1}^{u}(p)$, em ambos os casos, supor que $\mathcal{W}_{1}^{u}(p) \nsubseteq K$, implica na existência de um $t_{0} \in(0, \infty)$ que satisfaz:

(i) Se $t \in\left[0, t_{0}\right]$, então $l(t) \in K \cap \mathcal{W}_{1}^{u}(p)$.

(ii) Existe sequência $\left\{t_{n}\right\}_{n \in \mathbb{N}}, t_{n} \in\left(t_{0}, \infty\right)$, com $t_{n} \longrightarrow t_{0}^{+}$tal que $l\left(t_{n}\right) \notin K$.

Além disso, veja que a existência deste $t_{0}$ implica que, para todo $t \in\left(t_{0}, \infty\right), l(t) \notin K$. De fato, caso exista $t_{1}>t_{0}$ satisfazendo $l\left(t_{1}\right) \in K$, então existe um arco $l\left(\left[t_{0}, t_{1}\right]\right) \subset \mathcal{W}_{1}^{u}(p)$ intersectando o continuo $K$ nos pontos $l\left(t_{0}\right)$ e $l\left(t_{1}\right)$. Consequentemente, pelo Lema 2.2.2, $\left(\mathcal{W}^{u}(p) \cup K\right)^{c}$ iria possuir pelo menos duas componentes conexas, isto porque $\mathcal{W}^{u}(p) \subset \Sigma$. 
No entanto, como $\left(\mathcal{W}^{u}(p) \cup K\right) \subset \Sigma$, isto seria uma contradição com o fato de que o complementar de $\Sigma$ é conexo ou que interior de $\Sigma$ é vazio.

Defina $K_{0}=\mathcal{W}_{1}^{u}(p) \cap K$. Com a parametrização $l$ de $\mathcal{W}_{1}^{u}(p)$, segue-se que $K_{0}=$ $l\left(\left[0, t_{0}\right]\right)$. Veja que, para todo $j \in \mathbb{Z}$,

$$
h^{j}\left(K_{0}\right)=h^{j}\left(l\left(\left[0, t_{0}\right]\right)\right)=l\left(\left[0, t_{j}\right]\right) \text {, }
$$

onde $l\left(t_{j}\right)=h^{j}\left(l\left(t_{0}\right)\right)$. Desse modo, para todo $n \in \mathbb{N}$,

$$
l\left(\left[0, t_{0}\right]\right)=K_{0}=\bigcup_{j=0}^{n} h^{-j}\left(K_{0}\right) .
$$

Por outro lado, o Lema 4.3.1 nos mostra que existe $n_{0}>0$ tal que

$$
\mathcal{W}_{1}^{u}(p) \subset \bigcup_{j=0}^{n_{0}} h^{-j}(K) .
$$

Logo, como

$$
\bigcup_{j=0}^{n_{0}} h^{-j}\left(K_{0}\right)=\mathcal{W}_{1}^{u}(p) \cap \bigcup_{j=0}^{n_{0}} h^{-j}(K),
$$

tomando $n=n_{0}$, com as equações acima, chegamos que

$$
l\left(\left[0, t_{0}\right]\right)=\mathcal{W}_{1}^{u}(p)
$$

Mas isto é um absurdo, pois, como foi construído, $K_{0}=l\left(\left[0, t_{0}\right]\right) \subsetneq \mathcal{W}_{1}^{u}(p)$. Note que a contradição surgiu quando assumimos que $\mathcal{W}_{1}^{u}(p) \cap K \neq \emptyset$ e $\mathcal{W}_{1}^{u}(p) \nsubseteq K$. Portanto, nas condições do enunciado, se $K$ intersecta algum ramo de $\mathcal{W}^{u}(p)$, como $K$ é um fechado, então $K$ contém o fecho de tal ramo, provando assim a afirmação.

Observação 4.3.2. Com este Teorema, para $h \in \mathcal{H} \operatorname{com} \Sigma=\overline{\mathcal{W}^{u}(p)}$ e número de rotação $\rho(h, U) \in \mathbb{Q}^{c}$, então $K_{1}^{a}=K_{2}^{a}=\Sigma$ para todo a $\in \Sigma$ acessivel.

Corolário 4.3.1. Nas condições do Teorema 4.3.1, dado a $\in \Sigma$ um ponto acessível, vamos denotar por $\sigma_{a}^{m, n}$ um crosscut qualquer com extremos em $h^{n}(a)$ e $h^{m}(a)$. De modo semelhante, denotando por $D_{1}^{m, n}$ e $D_{2}^{m, n}$ as componentes conexas de $\left(\Sigma \cup \sigma_{a}^{m, n}\right)^{c}$ e definindo 


$$
K_{i}^{a_{m, n}}=\left.\partial D_{i}^{m, n}\right|_{\Sigma}
$$

pode-se repetir os argumentos do Teorema 4.3.1 e provar que se o número de rotação $\rho(h, U)$ é irracional, então

$$
K_{1}^{a_{m, n}}=K_{2}^{a_{m, n}}=\Sigma \text {, para quaisquer } m, n \in \mathbb{Z}
$$

Observação 4.3.3. Segue diretamente do Teorema 4.3.1 e do Corolário 4.3.1 que se existir algum ponto acessível $a \in \Sigma$ satisfazendo $K_{i}^{a_{m, n}} \subset \Sigma \backslash \mathcal{W}^{u}(p)$ para algum par de inteiros $m$ e $n$ e algum $i=1$ ou 2 , então o número de rotação $\rho(h, U) \in \mathbb{Q}$.

Quando $h$ é uma ferradura de Smale, o Teorema 4.2.1 mostrou que existe ponto periódico acessível de sela $q \in \Sigma$. Um ponto $q$ com estas características, possui a propriedade de que todos os pontos da variedade $\mathcal{W}^{u}(q)$ são acessíveis. Quando todos os pontos de uma variedade $\mathcal{W}^{u}(q)$ forem acessíveis, chamaremos tal variedade de variedade instável acessível.

Considere $h$ uma ferradura de Smale e $q$ um ponto periódico acessível. Como $\mathcal{W}^{u}(q)$ é uma variedade instável acessível, tomemos $a \in \mathcal{W}^{u}(q)$. Pelo Corolário 4.2.2, sabemos que $q$ ou é fixo ou tem período 2. Suponhamos que $q$ seja fixo, caso contrário, basta considerar $h^{2}$. Então, tomando qualquer crosscut $\sigma_{a}$ com extremos nos pontos acessíveis $a$ e $h(a)$, veja que para um $K_{i}$, digamos $K_{1}$,

$$
\partial K_{1}=\left.\mathcal{W}^{u}(q)\right|_{[a, h(a)]} \cup \sigma_{a}
$$

Isto implica que $p \notin K_{1}^{a} \cap K_{2}^{a}$. Tal fato motiva a próxima definição.

Definição 4.3.1. Considere $h \in \mathcal{H}$. Dado um ponto acessível $a \in \Sigma$, seja $\sigma_{a}^{m, n}$ um crosscut qualquer com extremos em $h^{n}(a)$ e $h^{m}(a)$. Diremos que o ponto a satisfaz a propriedade $K_{a}$ quando, para todo $n, m \in \mathbb{Z}$, tivermos $K_{1}^{a_{m, n}}=K_{2}^{a_{m, n}}=\Sigma$.

Desse modo, nos casos em que $h$ é uma ferradura de Smale, como o $\rho(h, U)$ é racional, segue do Corolário 4.2.1 que todo ponto acessível ou é um ponto periódico ou está na 
variedade instável de um ponto periódico acessível, resulta que não existe ponto acessível $a \in \Sigma$ satisfazendo a propriedade $K_{a}$.

Por outro lado, se $h$ não é ferradura de Smale, assumindo que o número de rotação $\rho(h, U)$ possa ser irracional, necessariamente, $h$ satisfaz a propriedade $K_{a}$ para todo ponto acessível $a \in \Sigma$. Além disso, não existem pontos acessíveis na variedade instável de qualquer ponto periódico de sela. 


\section{Referências Bibliográficas}

[AY92] K.T. Alligood, J.A. Yorke, Accessible saddles on fractal basin boundaries, Er- god. Th. Dynam. Sys. 12 (1992), 377-400.

[Bar93] M. Barge, Prime end rotation numbers associated with the Hénon maps, continuo theory and dynamical systems, Lecture Notes in Pure and Appl. Math., vol. 149, Dekker, New York, 1993, pp. 15?33.

[BaK98] M. Barge, K. Kuperberg, Periodic points from periodic prime ends, Proceedings of the 1998 Topology and Dynamics Conference (Fairfax, VA), vol. 23, 1998, 13-21.

[BG91] M. Barge, R.M. Gillette, Rotation and periodicity in plane separating continua, Ergodic Theory Dynam. Systems 11 (1991), no. 4, 619-631.

[BG92] M. Barge, R.M. Gillette, A fixed point theorem for plane separating continua, Topology Appl. 43 (1992), no. 3, 203-212.

[BS11] B. Gomes, S. Zanata Horseshoes for a Generalized Markus Yamabe Example, Qualitative Theory of Dynamical Systems, v. 1, p. 1-10, 2011.

[Ca13] C. Carathéodory, Uber die Begrenzung einfach zusammenhängender Gebiete, Math. Ann. 73 (1913) 323-370

[Ca81] Carr J. (1981) : Applications of centre manifold theory. Applied Mathematical Sciences 35. Springer-Verlag, New York-Berlin. 
[CL51] M.L. Cartwright, J.E. Littlewood, Some fixed point theorems, Annals of Math. 54 (1951), 1-37.

[COR10] L. Hernandez-Corbato, R. Ortega and Francisco R. Ruiz del Portal, Attractors with irrational rotation number, Math. Proc. Camb. Phil. Soc. 153 (2012), 59-77.

[De32] A. Denjoy, Sur les courbes definies par les équations différentielles à la surfade du tore. J. Math. Pores Appl. 11 (1932, 333-375).

[El08] L, Elon Lages, Análise Real - Volume 2, Coleção Matemática Universitária, Rio de Janeiro: IMPA, 2004.

[FC03] J. Franks and P. Le Calvez. Regions of instability for non-twist maps. Ergodic Theory Dynam. Systems, 23(1):111?141, 2003.

[KH99] A. Katok, B. Hasselblatt; Introduction to the moderm theory of Dynamical Systems, Cambridge University Press, 1999.

[KLN12] A. Koropecki, P. Le Calvez, M. Nassiri, Prime ends rotation numbers and periodic points, preprint, arXiv:1206.4707.

[KaHa95] Katok, A. and Hasselblatt, B., Introduction to the Modern Theory of Dynamical Systems, Encyclopedia Math. Appl., vol. 54, Cambridge: Cambridge Univ. Press, 1995.

[Go07] B. Gomes, A conjectura discreta de Markus-Yamabe, Tese de Doutorado, Universidade de São Paulo, 2007.

[Ha82] HARTMAN, P. Ordinary differential equations. Boston: Birkhouser, 1982.

[Is09] C. Isnard, Introdução à Medida e Integração, Projeto Elclides, 2ed. Rio de Janeiro: IMPA,2009.

[Ma82] J. Mather. Topological proofs os some purely tolological consequences of carathéodory's theory of prime ends. in Selected Studies. Eds. Th. M. Rassias and G. M. Rassias, pages 225-255, 1982. 
[Mat13] S. Matsumoto, Nontrivial attractor-repeller maps of $S^{2}$ and rotation numbers, preprint, arXiv:1302.4041.

[MePa77] de Melo, W.; Palis J. Introdução aos Sistemas Dinâmicos. Rio de Janeiro: IMPA, 1977

[OR11] R. Ortega, F.R. Ruiz del Portal, Attractors with vanishing rotation number, J. Eur. Math. Soc. 13 (2012), no. 6, 1569-1590.

[Pi82] D. PIXTON Planar homoclinic points, J. of Diff. Eq, 44 (1982), 365-382.

[Po52] H. Poincaré, Ouevres Complètes. Tome I, Gauthier - Villars, Paris, (1952), 137-1

[Po91] Ch. Pommerenke, Boundary behavior of conformal maps, Lectures Notes in Math. Springer-Verlag, 1991.

[Ro70] C. Robinson, Generic properties of conservative systems, i, ii. Amer. J. of Math, 92:562-603, 897-906, 1970.

[Ro73] C. Robinson, Clossing stable and unstable manifolds on the two sphere, Proc. A.M.S., 41 (1973), 299-303.

[Spa66] Spanier, E. Algebraic topology. Mc Graw-Hill, New York, 1966

[Oli87] F. Oliveira. On the generic existence of homoclinic points. Engod. The. e Dynam. Sys., 7:567-595, 1987.

[Ze73] Zehnder E. (1973) Homoclinic points near elliptic fixed points, Communications on Pure and Applied Mathematics 26, 131-182. 